TRANSACTIONS OF THE

AMERICAN MATHEMATICAL SOCIETY

Volume 187, Issue 1, 1974

\title{
SELFADJOINT ALGEBRAS OF UNBOUNDED OPERATORS. II(1)
}

\author{
BY
}

\author{
ROBERT T. POWERS $\left({ }^{2}\right)$
}

\begin{abstract}
Unbounded selfadjoint representations of *-algebras are studied. It is shown that a selfadjoint representation of the enveloping algebra of a Lie algebra can be exponentiated to give a strongly continuous unitary representation of the simply connected Lie group if and only if the representation preserves a certain order structure. This result follows from a generalization of a theorem of Arveson concerning the extensions of completely positive maps of $C *$-algebras. Also with the aid of this generalization of Arveson's theorem it is shown that an operator $\overline{\pi(A)}$ is affiliated with the commutant $\pi(Q)^{\prime}$ of a selfadjoint representation $\pi$ of a $*$-algebra $Q$, with $A=A * \in Q$, if and only if $\pi$ preserves a certain order structure associated with $A$ and $Q$. This result is then applied to tobtain a characterization of standard representations of commutative *-algebras in terms of an order structure.
\end{abstract}

Introduction. In this paper we continue our study of unbounded selfadjoint representations of *-algebras begun in a previous paper [5].

In [4] Nelson showed that there are selfadjoint representations of enveloping algebras of Lie algebras which cannot be exponentiated to give a strongly continuous unitary representation of the simply connected Lie group. In this same paper Nelson showed that a representation $\pi$ of the enveloping algebra of a Lie algebra can be exponentiated to give a unitary representation of the Lie group if a certain second order elliptic element $\Delta$ of the enveloping algebra is represented by an operator $\pi(\Delta)$ which has a dense set of analytic vectors. In this paper we show in Theorem 4.5 that a selfadjoint representation of the enveloping algebra of a Lie algebra can be exponentiated to give a strongly continuous unitary representation of the simply connected Lie group if and only if the representation preserves a certain order structure described in §IV of this paper.

The basic idea of the proof of this result is as follows. Suppose $G$ is a connected simply connected $\mathrm{Lie}$ group and $\mathcal{L}(G)$ its Lie algebra. Let $\mathbb{Q}(G)$ be the universal enveloping algebra of $\mathscr{L}(G)$, the algebra of all polynomials in elements $X \in \mathscr{L}(G)$ modulo the commutation relations of the Lie algebra. We construct a *algebra $\mathfrak{B}_{u}$ which is generated by $\mathfrak{Q}(G)$ and unitary elements $U(g)$ for each $g \in G$,

Received by the editors January 19, 1973.

AMS (MOS) subject classifications (1970). Primary 46L99; Secondary 47D20, 22 E45.

(1) Work supported in part by the National Science Foundation.

(2) Alfred P. Sloan Foundation Fellow.

Copyright $\odot 1974$, American Mathematical Society 
which form a representation of $G$ and act on $\mathscr{Q}(G)$ by *-automorphisms, i.e. $U(g)^{*} A U(g)=a_{g}(A)$ for each $A \in \mathbb{Q}(G)$ and $g \in G$ where $g \rightarrow a_{g}$ is a representation of $G$ by *-automorphisms of $\mathfrak{Q}(G)$. In essence we have that a selfadjoint representation $\pi$ of $\mathscr{Q}(G)$ can be exponentiated to give a strongly continuous unitary representation of $G$ if and only if $\pi$ can be extended from a representation of $\mathscr{Q}(G)$ to a representation of $B_{u}$. Then the problem is one of extending a representation $\pi$ of a *-algebra $\mathfrak{Q}$ to a larger *-algebra $B$.

Every bounded *-representation of a *-algebra $\mathbb{Q}$ can be expressed as a direct sum of cyclic representations each of which is characterized by a state of $\mathfrak{Q}$. In view of this fact, the problem of extending a bounded *-representation $\pi$ of a *algebra $\mathbb{C}$ to a larger *-algebra $\mathbb{B}$ can be expressed in terms of the problem of extending states of a *algebra $\mathbb{A}$ to a larger *-algebra $B$. This problem can be analysed with the aid of theorems such as the Hahn-Banach theorem. For unbounded *-representations of a *-algebra it is not necessarily true that a ${ }^{*}$-representation can be expressed as a direct sum of cyclic representations. For this reason in considering the problem of extending unbounded *-representations we use the tool of completely positive maps and their connection with *-representations rather than the less complicated tool of states.

In \$II of this paper we discuss Stinespring's work in [6] on completely positive maps and their relation with ${ }^{*}$-representations of $C^{*}$-algebras. We make the minor modifications necessary to adopt completely positive maps to unbounded *-representations of *-algebras.

In [1] Arveson proved an extension theorem for completely positive maps on $C^{*}$-algebras. In \$III of this paper we prove a generalization of Arveson's theorem which is applicable to unbounded ${ }^{*}$-representations. This generalization shows that the result of Arveson's theorem is actually quite independent of topology and algebraic structure.

In SIV of this paper we apply the generalization of Arveson's theorem to selfadjoint representations of the enveloping algebras of Lie algebras as discussed earlier in the introduction. In $\$ \mathrm{~V}$ we apply the generalization of Arveson's theorem to the question of determining when a hermitian operator $\overline{\pi(A)}$ is affiliated with the commutant $\pi(\mathfrak{Q})^{\prime}$ of a selfadjoint representation $\pi$ of a *algebra. In Theorem 5.3 we give a necessary and sufficient condition that $\overline{\pi(A)}$ be affiliated with $\pi(\mathcal{Q})^{\prime}$ where $A=A^{*} \in \mathbb{Q}$ and $\pi$ is a selfadjoint representation of $\mathbb{Q}$ on a Hilbert space. The condition is expressed in terms of an order structure of $\mathbb{Q}$.

We then use Theorem 5.3 to characterize standard representations fo commutative *-algebras. A standard representation $\pi$ of a commutative *algebra $Q$ is a selfadjoint representation such that if $A, B \in \mathbb{Q}$ and $A=A^{*}, B=B^{*}$, then $\pi(A)$ and $\pi(B)$ are essentially selfadjoint and have mutually commuting spectral projections. In Theorem 5.6 we show that a selfadjoint representation $\pi$ of a commutative *-algebra with unit is standard if and only if and only if $\pi$ preserves a certain order 
structure. Theorem 5.6 is a generalization of Theorem 7.3 of [5], in that in [5] we worked with strongly cyclic representations and in this paper we work with arbitrary selfadjoint representations.

In $\$ I$ of this paper we review the basic notation and results of [5].

The author would like to thank Dr. Carl Kaysen for hospitality shown the author during his visit to the Institute for Advanced Study where most of the research for this paper was done. The author would like to thank Professor Oscar Lanford for several helpful discussions during the research for this paper.

I. Notation, definitions and some previous results. In this section we review some of the definitions and simplest results concerning unbounded *-representations of *-algebras on Hilbert space and refer to [5] for further details, references and proofs of the results stated here. We begin with a quick review of the notation we will use to describe a single unbounded operator on a Hilbert space. In this paper we work exclusively with Hilbert spaces over the complex numbers. We use the physicists' inner product $(f, g)$ which is linear in $g$ and conjugate linear in $f$.

A bounded or unbounded operator $A$ on a Hilbert space $\mathcal{H}$ defined on a linear manifold $\mathcal{D}(A)$ (called the domain of $A$ ) is a linear mapping of $\mathscr{D}(A)$ into $\mathcal{H}$. Unless otherwise stated it is assumed that $\mathscr{D}(A)$ is dense in $\mathcal{H}$. If $A$ and $B$ are operators in $\mathcal{H}$ with domains $\mathscr{D}(A)$ and $\mathscr{D}(B)$ we say $A$ extends $B$, denoted $A \supset B$, if $\mathscr{D}(A) \supset \mathcal{D}(B)$ and $A f=B f$ for all $f \in \mathscr{D}(B)$. An operator $A$ is said to be closable if it has a closed extension. If $A$ is closable we denote by $\bar{A}$ the minimal closed extension of $A$.

If $A$ is an operator with dense domain we denote by $A^{*}$ the hermitian adjoint of $A$. As is well known, $A$ is closable if and only if $A^{*}$ is densely defined, and if $A$ is closable then $\bar{A}=A^{* *}$. An operator $A$ is hermitian if $A^{*} \supset A$. An operator $A$ is selfadjoint if $A=A^{*}$, and an operator $A$ is essentially selfadjoint if $\bar{A}=A^{*}$.

If $A$ is a hermitian operator we denote by $\mathscr{D}_{+}$and $\mathscr{D}_{-}$the deficiency spaces of $A$ defined by the relations

$$
\begin{aligned}
& \mathscr{D}_{+}=\{\text {Range }(A+i I)\}^{\perp}=\left\{f \in \mathscr{D}\left(A^{*}\right) ; A^{*} f=i f\right\}, \\
& \mathscr{D}_{-}=\{\text {Range }(A-i I)\}^{\perp}=\left\{f \in \mathcal{D}\left(A^{*}\right) ; A^{*} f=-i f\right\},
\end{aligned}
$$

where $\{S\}^{\perp}$ denotes the orthogonal complement of $S$. A useful result of von Neumann states that a hermitian operator $A$ is essentially selfadjoint if and only if $D_{+}=$ $D_{-}=\{0\}$.

In this paper we will be concerned with unbounded *-representations of *-algebras. $A^{*}$-algebra $\mathfrak{Q}$ is an algebra over the complex numbers with a *operation satisfying: 
(i) $A^{* *}=A$,

(ii) $(a A+B)^{*}=\bar{\alpha} A^{*}+B^{*}$,

(iii) $(A B)^{*}=B^{*} A^{*}$,

for all $A, B \in \mathbb{Q}$ and complex numbers $a$.

All algebras in this section will bave a unit denoted by $I$.

Definition 1.1. A representation $\pi$ of an algebra $\mathcal{A}$ on a Hilbert space $\mathcal{H}$ is a mapping of $\mathfrak{Q}$ into linear operators all defined on a common linear manifold $\mathscr{D}(\pi)$ which is dense in $\mathcal{H}$, and $\pi$ satisfies the conditions, $\pi(I)=I$, the unit operator on $\mathcal{H}$,

(i) $\pi(a A+B) f=a \pi(A) f+\pi(B) f$ for all $A, B \in \mathbb{Q}, f \in \mathscr{D}(\pi)$ and complex numbers $a$.

(ii) $\pi(A) \mathscr{D}(\pi) \subset \mathscr{D}(\pi)$ for all $A \in \mathbb{Q}$ and $\pi(A) \pi(B) f=\pi(A B) f$ for all $A, B \in \mathbb{Q}$ and $f \in \mathcal{D}(\pi)$.

The linear manifold $\mathscr{D}(\pi)$ is called the domain of $\pi$.

Definition 1.2. A representation $\pi$ of a ${ }^{*}$-algebra $\mathfrak{A}$ on a Hilbert space $\mathcal{H}$ is said to be hermitian or a ${ }^{*}$-representation if

(iii) $(f, \pi(A) g)=\left(\pi\left(A^{*}\right) f, g\right)$ for all $f, g \in \mathscr{D}(\pi)$ and $A \in \mathbb{Q}$, i.e. $\pi(A)^{*} \supset \pi\left(A^{*}\right)$ for all $A \in \mathbb{Q}$.

We remark that a representation $\pi$ is hermitian if and only if $\pi(A)$ is hermitian for every hermitian $A \in \mathbb{Q}$ (i.e. $A=A^{*}$ ).

Definition 1.3. If $\pi_{1}$ and $\pi_{2}$ are representations of an algebra $\mathfrak{Q}$ on a Hilbert space $\mathcal{H}$ we say $\pi_{1}$ is an extension of $\pi_{2}$, denoted $\pi_{1} \supset \pi_{2}$, if $\mathcal{D}\left(\pi_{1}\right) \supset \mathcal{D}\left(\pi_{2}\right)$ and $\pi_{1}(A) \supset \pi_{2}(A)$ for all $A \in \mathbb{Q}$.

If $\pi$ is a representation of an algebra $\mathfrak{A}$ on a Hilbert space $\mathcal{H}$ with domain $\mathscr{D}(\pi)$, there is a natural induced topology on $\mathscr{D}(\pi)$. This topology is defined as follows. Suppose $S$ is a finite set of elements of $\mathcal{Q}$. We define the seminorm $\|\cdot\|_{S}$ on $\mathcal{D}(\pi)$ by the relation

$$
\|f\|_{S}=\sum_{A \in S}\|\pi(A) f\|
$$

where $\|\pi(A) f\|$ is the Hilbert space norm of $\pi(A) f$. Note $S \supset S^{\prime}$ implies $\|f\|_{S} \geq$ $\|f\|_{S^{\prime}}$ for all $f \in \mathscr{D}(\pi)$. We define the induced topology on $\mathscr{D}(\pi)$ as the topology generated by the neighborhoods,

$$
N(f ; S, \epsilon)=\left\{g \in \mathscr{D}(\pi) ;\|f-g\|_{S}<\epsilon\right\} .
$$

Note $\pi(A)$ is a continuous mapping of $\mathscr{D}(\pi)$ into $\mathscr{D}(\pi)$ in the induced topology for each $A \in \mathbb{Q}$.

Definition 1.4. Suppose $\pi$ is a representation of an algebra $\mathfrak{Q}$ on a Hilbert space $\mathcal{H}$. We say a set $S \subset \mathscr{D}(\pi)$ is strongly dense in $\mathscr{D}(\pi)$ if $S$ is dense in $\mathscr{D}(\pi)$ in the induced topology.

Just as there is a notion of a closed operator there is a notion of a closed representation. 
Definition 1.5. We say $\pi$ is a closed representation of $\mathscr{Q}$ if $\mathcal{D}(\pi)$ is complete in the induced topology.

Every hermitian operator is closable. The analogous result holds for *-representations.

Lemma 1.6. Suppose $\pi$ is $a^{*}$-representation of $a^{*}$-algebra $\mathbb{Q}$ on a Hilbert space $\mathcal{H}$. Then there is a unique minimal closed extension $\hat{\pi}$ of $\pi$. Furthermore the domain of $\tilde{\pi}$ is given by

$$
\mathscr{D}(\tilde{\pi})=\bigcap_{A \in Q} \bar{D} \overline{(\pi(A \cdot))}
$$

and $\tilde{\pi}(A) f=\overline{\pi(A)} f$ for all $A \in \mathbb{Q}$ and $f \in \mathscr{D}(\pi)$.

Hereafter, we denote by $\tilde{\pi}$ the minimal closed extension of the ${ }^{*}$-representation $\pi$. Note we always have that $\mathscr{D}(\pi)$ is strongly dense in $\mathscr{D}(\tilde{\pi})$ for any *-representation $\pi$.

Definition 1.7. Suppose $\pi$ is a ${ }^{*}$-representation of a ${ }^{*}$-algebra $\mathfrak{Q}$ on a Hilbert space $\mathcal{H}$. The commutant of $\pi(\mathfrak{Q})$, denoted by $\pi(\mathfrak{Q})^{\prime}$, consists of all bounded operators $C$ on $\mathcal{H}$ such that

$$
(f, C \pi(A) g)=\left(\pi\left(A^{*}\right) f, C g\right)
$$

for all $f, g \in \mathscr{D}(\pi)$ and $A \in \mathbb{Q}$.

If $\pi(\mathfrak{Q})$ is a *algebra of bounded operators then $\pi(\mathfrak{Q})^{\prime}$ is simply the commutant of $\pi(\mathfrak{Q})$ defined in the usual manner. The above definition is the one used in the Wightman formulation of quantum field theory. This definition is essentially the weakest definition, i.e. this definition gives the biggest commutant.

One can easily check that the commutant $\pi(\mathfrak{Q})^{\prime}$ of a ${ }^{*}$-representation $\pi$ has the following properties:

(i) $\pi(\mathfrak{Q})^{\prime}$ is a complex linear manifold.

(ii) $\pi(\mathfrak{Q})^{\prime}$ is symmetric, i.e. $C \in \pi(\mathfrak{Q})^{\prime}$ implies $C^{*} \in \pi(\mathfrak{Q})^{\prime}$.

(iii) $\pi(\mathfrak{Q})^{\prime}$ is closed in the weak operator topology.

(iv) If $\tilde{\pi}$ is the closure of $\pi$ then $\tilde{\pi}(\mathfrak{Q})^{\prime}=\pi(\mathfrak{Q})^{\prime}$.

In general $\pi(\mathfrak{Q})^{\prime}$ is not an algebra. It is shown in [5] that if $\mathfrak{Q}$ is the free commutative algebra on one hermitian generator (i.e. $\mathbb{Q}$ consists of all polynomials $P=a_{0} I+a_{1} A_{0}+\cdots+a_{n} A_{0}^{n}$ with complex coefficients) and $\pi$ is a *-representation of $\mathfrak{Q}$ on a Hilbert space $\mathcal{H}$, then $\pi(\mathfrak{A})^{\prime}$ is an algebra if and only if $\pi\left(A_{0}\right)$ is essentially selfadjoint.

Just as there is the notion of the hermitian adjoint of an operator there is the notion of the hermitian adjoint of a representation.

Lemma 1.8. Suppose $\pi$ is a representation of $a^{*}$-algebra $\mathfrak{Q}$ on a Hilbert space H. Let 
and let

$$
\mathcal{D}\left(\pi^{*}\right)=\bigcap_{A \in \mathcal{R}} \mathfrak{D}\left(\pi(A)^{*}\right)
$$

$$
\pi^{*}(A)=\pi\left(A^{*}\right)^{*} \mid \mathcal{D}\left(\pi^{*}\right) \text { for all } A \in \mathbb{Q} \text {. }
$$

Then $\pi^{*}$ is a closed representation of $\mathfrak{Q}$ on $\mathcal{H}$ with domain $\mathfrak{D}\left(\pi^{*}\right)$. If $\pi$ is a * representation of $\mathbb{Q}$ then $\pi^{*} \supset \pi$.

We call $\pi^{*}$ the hermitian adjoint of $\pi$. If $\pi$ is hermitian, $\pi^{*}$ may fail to be hermitian just as the hermitian adjoint $A^{*}$ of a hermitian operator $A$ may fail to be hermitian. Just as for operators, one can show that every hermitian extension of a $*$-representation $\pi$ is a hermitian restriction of $\pi^{*}$. In fact, if $\pi$ and $\pi_{1}$ are hermitian and $\pi_{1} \supset \pi$ then $\pi^{*} \supset \pi_{1}^{*} \supset \pi_{1} \supset \pi^{*}$

Although it is not discussed in [5] we remark that one can show the following properties of $\pi^{* *}$ for ${ }^{*}$-representations $\pi$. If $\pi$ is a *-representation of a ${ }^{*}$-algebra $\mathfrak{Q}$ on a Hilbert space $\mathcal{H}$ then $\pi^{* *}$ is a closed hermitian extension of $\pi$. Unlike the case for single operators, there are examples of closed *-representations $\pi$ such that $\pi^{* *}$ is a proper extension of $\pi$. One can show $\pi^{* * *}=\pi^{*}$ and hence $\pi^{* * * *}=\pi^{* *}$ for all ${ }^{*}$-representations $\pi$. Hence, no new representations are obtained after taking two hermitian adjoints.

Definition 1.9. We say a ${ }^{*}$-representation $\pi$ of a ${ }^{*}$-algebra $\mathfrak{Q}$ is selfadjoint if $\pi=\pi^{*}$. We say $\pi$ is essentially selfadjoint if the closure $\tilde{\pi}$ is selfadjoint.

It follows from Lemma 1.8 that a hermitian representation is selfadjoint if and and only if $\mathscr{D}(\pi)=\mathscr{D}\left(\pi^{*}\right)$.

Lemma 1.10. Suppose $\pi$ is $a^{*}$-representation of $a^{*}$-algebra $\mathfrak{Q}$ on a Hilbert

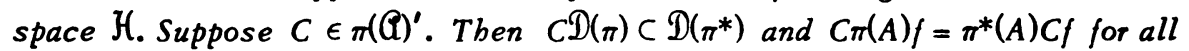
$A \in \mathbb{Q}$ and $f \in \mathscr{D}(\pi)$.

Lemma 1.11. Suppose $\pi$ is a selfadjoint representation of a *algebra. Then, the commutant $\pi(\mathfrak{Q})^{\prime}$ is a von Neumann algebra. Furthermore, for each $C \in \pi(\mathfrak{Q})^{\prime}$ we bave $C \mathscr{D}(\pi) \subset \mathscr{D}(\pi)$ and $C \pi(A) f=\pi(A) C f$ for all $A \in \mathbb{Q}$ and $f \in \mathscr{D}(\pi)$.

We turn next to the problem of reducing subspaces. If $\pi$ is a representation of an algebra $\mathcal{A}$ on a Hilbert space $\mathcal{H}$ and $\mathbb{M}$ is a linear manifold contained in $\mathcal{D}(\pi)$ we say $\mathbb{R}$ reduces $\pi$ if $\pi(A) \mathbb{M \subset} \mathbb{M}$ for all $A \in \mathbb{Q}$. We denote by $\pi \mid m$ the representation $\pi$ restricted to $\mathbb{R}$. If $\pi$ is a ${ }^{*}$-representation of a *algebra $\mathbb{Q}$ and $\mathbb{M}$ is a reducing subspace then $\pi \mid \mathbb{M}$ is a $*$-representation of $\mathbb{Q}$ on $\bar{M}$.

For a bounded *-representation of a ${ }^{*}$-algebra $\mathfrak{Q}$ for each reducing subspace $\mathbb{M}$ the hermitian projection $E$ onto the closure of $\mathbb{M}$ is in the commutant $\pi(\mathfrak{Q})^{\prime}$. This is not true in general for unbounded *-representations. An example is given in [s]. 
We say a reducing subspace $\Re$ is selfadjoint if $\pi \mid M$ is a selfadjoint representation.

Theorem 1.12. Suppose $\pi$ is a selfadjoint representation of $a^{*}$-algebra $\mathbb{Q}$ on a Hilbert space $\mathcal{H}$. Suppose $E \in \pi(\mathfrak{Q})^{\prime}$ is a bermitian projection. Let $\mathbb{M}=E \mathfrak{D}(\pi)$. Then $M$ reduces $\pi$ and the restriction $\pi \mid \mathcal{M}$ is selfadjoint.

Conversely, suppose $\mathbb{M} \subset \mathscr{D}(\pi)$ is a reducing subspace for $\pi$ and $\pi \mid \mathbb{M}$ is selfadjoint. Then, the bermitian projection $E_{\mathfrak{D} /}$ onto the closure of $\mathbb{M}$ is in the commutant $\pi(\mathfrak{Q})^{\prime}$.

Hence, for selfadjoint representations there is a one-to-one correspondence between bermitian projections in $\pi(\mathfrak{Q})^{\prime}$ and selfadjoint reducing subspaces.

We conclude this section with some remarks about the notion of closed operators affiliated with a von Neumann algebra. We say a closed operator $A$ is affiliated with a von Neumann algebra $R$ if $U^{*} A U=A$ for all unitary $U \in R^{\prime}$ the commutant of $R$, i.e. $U \mathscr{D}(A) \subset \mathscr{D}(A)$ for all unitary $U \in R^{\prime}$ and $U^{*} A U f=A f$ for all $f \in \mathscr{D}(A)$. It follows that a closed operator $A$ is affiliated with $R$ if and only if $A^{*}$ is affiliated with $R$. If $\{E(\lambda) ;-\infty<\lambda<+\infty\}$ is the spectral resolution of a selfadjoint operator $A$ then $A$ is affiliated with a von Neumann algebra $R$ if and only if $E(\lambda) \in R$ for all real $\lambda$. If $A$ is a closed hermitian operator affiliated with $R$ and $E_{ \pm}$are the hermitian projections onto the deficiency spaces $\mathfrak{D}_{ \pm}$then $E_{ \pm} \in R$.

If $\pi$ is a selfadjoint representation of a *algebra $\mathfrak{Q}$ we denote by $\pi(\mathfrak{Q})^{\prime \prime}$ the commutant of $\pi(\mathfrak{Q})^{\prime}$, i.e. $\pi(\mathfrak{Q})^{\prime \prime}=\left(\pi(\mathfrak{Q})^{\prime}\right)^{\prime}$. It follows almost immediately from Lemma 1.11 that if $\pi$ is a selfadjoint representation of a ${ }^{*}$-algebra $\mathfrak{Q}$ then $\overline{\pi(A)}$ and $\pi(A)^{*}$ are affiliated with $\pi(\mathfrak{Q})^{\prime \prime}$ for all $A \in \mathbb{Q}$.

II. Completely positive maps. In this section we discuss the application of a theorem of Stinespring concerning completely positive maps of $C^{*}$-algebras to unbounded *-representations of *-algebras. The generalization of Stinespring's theorem to the unbounded case presents no real difficulties.

For a discussion of Stinespring's theorem we refer to Stinespring's paper [6], and for a short account and an outline of a simplified proof we refer to the first section of Arveson's paper [1]. A similar chain of results expressed in a different terminology can be found in a paper of Sz.-Nagy [7].

Definition 2.1. Suppose $Q$ is a ${ }^{*}$-algebra with unit $I$. A cone $Q$ in the real linear space of hermitian elements of $\mathscr{Q}$ is said to be algebraically admissible if

(i) $Q$ is a cone in that if $A, B \in Q$ and $\lambda, \mu \geq 0$ then $\lambda A+\mu B \in Q$.

(ii) $I \in Q$.

(iii) If $A \in Q$ and $B \in \mathbb{Q}$ then $B^{*} A B \in Q$.

If $\mathcal{Q}$ is a *algebra with identity and $Q$ is an algebraically admissible cone for $Q$ we say a state $\omega$ is strongly positive (with respect to $Q$ ) if $\omega(A) \geq 0$ for 
all $A \in Q$. We say a *-representation $\pi$ of $\mathfrak{Q}$ is strongly positive (with respect to $Q$ ) if $(f, \pi(A) f) \geq 0$ for all $A \in Q$ and $f \in \mathscr{D}(\pi)$.

Suppose $\mathfrak{Q}$ is a *algebra with unit. We denote by $M(\mathfrak{Q})$ the *-algebra of all finite matrices with entries in $\mathfrak{Q}$. Each element $\left\{A_{i j}\right\} \in M(\mathfrak{Q})$ is an array of elements $A_{i j} \in \mathbb{Q}$ for $i, j=1,2, \ldots$, where $A_{i j} \neq 0$ for only finitely many pairs of indices $(i, j)$. Addition, multiplication and the *-operation are defined in the obvious manner, i.e.

if $a\left\{x_{i j}\right\}+\left\{y_{i j}\right\}=\left\{z_{i j}\right\}$ then $z_{i j}=\alpha x_{i j}+y_{i j}$,

if $\left\{x_{i j}\right\}\left\{y_{i j}\right\}=\left\{z_{i j}\right\}$ then $z_{i j}=\sum_{k=1}^{\infty} x_{i k} y_{k j}$,

if $\left\{x_{i j}\right\}^{*}=\left\{y_{i j}\right\}$ then $y_{i j}=\left(x_{j i}\right)^{*}$.

We define an algebraically admissible cone for $M(\mathfrak{Q})$ in the same way as it was done for *algebras with a unit. We say a cone $Q_{m}$ in the real linear space of hermitian elements of $M(\mathfrak{Q})$ is algebraically admissible if

(i) $Q_{m}$ is a cone in that if $\left\{A_{i j}\right\},\left\{B_{i j}\right\} \in Q_{m}$ and $\lambda, \mu \geq 0$ then $\lambda\left\{A_{i j}\right\}+$ $\mu\left\{B_{i j}\right\} \in Q_{m}$.

(ii) If $\left\{A_{i j}\right\} \in Q$ and $\lambda \geq 0$ and $\left\{B_{i j}\right\} \in M(\mathbb{Q})$ then $\left\{B_{i j}\right\} *\left\{A_{i j}\right\}\left\{B_{i j}\right\}+$ $\lambda\left\{B_{i j}\right\} *\left\{B_{i j}\right\} \in Q_{m}$.

If $Q$ is an admissible cone for a ${ }^{*}$-algebra $\mathcal{Q}$ with unit and $Q_{m}$ is an admissible cone for $M(\mathbb{Q})$ we say $Q$ and $Q_{m}$ are compatible if $\left\{A \delta_{1 i} \delta_{1 j}\right\} \in Q_{m}$ if and only if $A \in Q$. The reader may easily verify that if $Q$ is an algebraically admissible cone for a ${ }^{*}$-algebra $\mathfrak{Q}$ then the maximal admissible cone $Q_{m}$ for $M(\mathfrak{Q})$ which is compatible with $A$ is given by

$$
\begin{aligned}
Q_{m}=\left\{\left\{A_{i j}\right\}=\left\{A_{i j}\right\}^{*} \in M(\mathfrak{Q}) ; \sum_{i j=1}^{n} A_{i}^{*} A_{i j} A_{j} \in Q\right. \\
\\
\left.\qquad \text { for all } A_{i} \in \mathbb{Q}, i=1, \ldots, n, n=1,2, \ldots\right\} .
\end{aligned}
$$

Next we define completely strongly positive maps of *-algebras. In our definition we use the following notation. Suppose $\mathcal{K}$ is a complex linear vector space. We denote by $\mathscr{B}(\mathcal{K})$ the space of all (conjugate linear-linear) bilinear forms on $\mathcal{K}$. Each element $\phi \in \mathfrak{B}(\mathcal{K})$ is a mapping of $\mathcal{K} \times \mathcal{K}$ into the complex numbers. The complex number associated with the pair $\{f, g\} \in \mathcal{K} \times \mathcal{K}$ by $\phi$ is denoted by $\langle f|\phi| g\rangle$. The form $\langle f|\phi| g\rangle$ is linear in $g$ and conjugate linear in $f$.

Definition 22. Suppose $\mathfrak{Q}$ is a *-algebra with unit and $Q_{m}$ is an algebraically admissible cone for $M(\mathfrak{Q})$. Suppose $\mathcal{K}$ is a complex linear vector space. A completely strongly positive map of $\mathfrak{Q}$ on $\mathcal{K}$ (with respect to $Q_{m}$ ) is a linear map $\phi$ of $\mathbb{Q}$ into $B(\mathcal{K})$ with the property that if $\left\{A_{i j}\right\} \in Q_{m}$ then

for all $f_{i} \in \mathcal{K}, i=1,2, \ldots$.

$$
\sum_{i j=1}^{\infty}\left\langle f_{i}\left|\phi\left(A_{i j}\right)\right| f_{j}\right\rangle \geq 0
$$


A map $\phi$ is a completely positive map of $\mathfrak{Q}$ on $\mathcal{K}$ if

$$
\sum_{i j=1}^{n}\left\langle f_{i}\left|\phi\left(A_{i}^{*} A_{j}\right)\right| f_{j}\right\rangle \geq 0
$$

for all $f_{i} \in \mathcal{K}, A_{i} \in \mathbb{Q}, i=1, \ldots, n$ and $n=1,2, \ldots$.

Note that in the infinite sum in the above definition there are only a finite number of nonzero terms since $A_{i j} \neq 0$ for only finitely many pairs of indices. We now state Stinespring's theorem for completely strongly positive maps.

Theorem 2.3 (Generalized Stinespring). Suppose $\mathfrak{Q}$ is a *algebra with unit and $Q_{m}$ is an algebraically admissible cone for $M(\mathfrak{Q})$. Suppose $\mathcal{K}$ is a complex linear vector space and $\phi$ is a completely strongly positive map (with respect to $Q_{m}$ ) of $\mathfrak{Q}$ on $\mathcal{K}$. Then there is a closed completely strongly positive (with respect to $Q_{m}$ ) *-representation of $\mathfrak{Q}$ on a Hilbert space $\mathcal{H}$ and a linear map $V$ of $\mathcal{K}$ into $\mathfrak{D}(\pi)$ such that

$$
\langle f|\phi(A)| g\rangle=(V f, \pi(A) V g)
$$

for all $f, g \in \mathcal{K}$ and $A \in \mathbb{Q}$ and $\{\pi(\mathfrak{Q}) V \mathcal{K}\}$ is strongly dense in $\mathscr{D}(\pi)$. Furthermore the representation $\pi$ is determined by the above requirements up to unitary equivalence.

Proof. For the proof of this theorem we will follow Arveson's outline of a simplified proof of Stinespring's theorem with the slight modification necessary for our case.

We begin by defining a bilinear form on the tensor product space $\mathbb{Q} \otimes \mathcal{K}$ by the relation if $F=\sum_{i=1}^{n} A_{i} \otimes f_{i}$ and $G=\sum_{j=1}^{m} B_{j} \otimes g_{j}$ with $A_{i}, B_{j} \in \mathbb{Q}$ and $f_{i}, g_{j} \in \mathcal{K}$ for $i=1, \ldots, n, j=1, \ldots, m$, then

$$
(F, G)=\sum_{i, j=1}^{n m}\left\langle f_{i}\left|\phi\left(A_{i}^{*} B_{j}\right)\right| g_{j}\right\rangle .
$$

The fact that $\phi$ is completely positive insures that this inner product is positive semidefinite on $\mathbb{Q} \otimes \mathcal{K}$. Let $N=\{F \in \mathbb{Q} \otimes \mathcal{K} ;(F, F)=0\}$. We define the operator $\pi^{\prime}(A)$ on $\mathbb{Q} \otimes \mathcal{K}$ for each $A \in \mathbb{Q}$ by the relation

$$
\pi^{\prime}(A) \sum_{i=1}^{n} A_{i} \otimes f_{i}=\sum_{i=1}^{n} A A_{i} \otimes f_{i^{\bullet}}
$$

Since $\phi$ is completely positive we have, for each $F=\sum_{i=1}^{n} A_{i} \otimes f_{i} \in \mathbb{Q} \otimes \mathcal{K}$,

$$
\omega(A)=\left(F, \pi^{\prime}(A) F\right)=\sum_{i, j=1}^{n}\left\langle f_{i}\left|\phi\left(A_{i}^{*} A A_{j}\right)\right| f_{j}\right\rangle
$$

is a positive linear functional on $\mathbb{Q}$ (i.e. $\omega\left(A^{*} A\right) \geq 0$ for all $A \in \mathfrak{Q}$ ). From the generalized Schwartz's inequality it follows that $|\omega(A)|^{2} \leq \omega(I) \omega\left(A^{*} A\right)$ for all $A \in \mathbb{Q}$. Hence if $\omega(I)=(F, F)=0$ it follows that $\omega\left(A^{*} A\right)^{-}=\left(\pi^{\prime}(A) F, \pi^{\prime}(A) F\right)=0$ 
for all $A \in \mathbb{Q}$. Therefore, $\pi^{\prime}(A)$ maps $N$ into itself for all $A \in \mathbb{Q}$.

Let $\mathfrak{D}$ be the quotient space $(\mathscr{Q} \otimes \mathcal{K}) / N$ of equivalence classes of $\mathbb{Q} \otimes \mathcal{K}$ modulo $N$. We denote by $[F] \in \mathscr{D}$ the equivalence class of all $F^{\prime} \in \mathbb{Q} \otimes \mathbb{K}$ such that $F-F^{\prime} \in N$. Since $\pi^{\prime}(A)$ maps $N$ into itself, the mapping $\pi_{1}(A)$ defined by $\pi_{1}(A)[F]=\left[\pi^{\prime}(A) F\right]$ is well defined on $\mathfrak{D}$ for all $A \in \mathbb{Q}$.

Let $\mathcal{H}$ be the Hilbert space obtained upon the completion of $\mathfrak{D}$ with respect to its inner product $\left([F],\left[F^{\prime}\right]\right)=\left(F, F^{\prime}\right)$. We consider $\mathfrak{D}$ as a dense subset of $\mathcal{H}$. Clearly, $\pi_{1}$ is a ${ }^{*}$-representation on $\mathcal{H}$ with domain $\mathcal{D}$. Let $\pi=\pi_{1}$ be the closure of $\pi$. We have $\mathfrak{D}$ is strongly dense in $\mathscr{D}(\pi)$.

We define a linear mapping $V$ of $\mathcal{K}$ in $\mathscr{D} \subset \mathscr{D}(\pi)$ by the relation $V f=[I \otimes f]$. A straightforward computation shows that

$$
\langle f|\phi(A)| g\rangle=\left(V f, \pi(A) V_{g}\right)
$$

for all $f, g \in \mathcal{K}$ and $A \in \mathbb{Q}$. We have $\{\pi(\mathfrak{Q}) V \mathcal{K}\}$ is strongly dense in $\mathcal{D}(\pi)$.

Next we show that $\pi$ is completely strongly positive. Suppose $\left\{A_{i j}\right\} \in Q_{m} \subset M(\mathscr{Q})$. Let $n$ be the smallest integer so that $A_{i j}=0$ if either $i>n$ or $j>n$. Let $r$ be any positive integer and let

$$
F_{i}=\sum_{k=1}^{r} \pi\left(B_{i k}\right) V f_{i k}
$$

with $B_{i k} \in \mathbb{Q}$ and $f_{i k} \in \mathcal{K}$ for $i=1, \ldots, n$ and $k=1, \ldots, r$. We have that

$$
\begin{aligned}
\sum_{i j=1}^{n}\left(F_{i}, \pi\left(A_{i j}\right) F_{j}\right) & =\sum_{i j k l}\left(\pi\left(B_{i k}\right) V f_{i k}, \pi\left(A_{i j}\right) \pi\left(B_{j l}\right) V f_{j l}\right) \\
& =\sum_{i j k l}\left\langle f_{i k}\left|\phi\left(B_{i k}^{*} A_{i j} B_{j l}\right)\right| f_{j l}\right\rangle \cdot
\end{aligned}
$$

It follows from the fact that $\left\{A_{i j}\right\} \in Q_{m}$ and the properties of admissible cones in $M(\mathfrak{Q})$ that the matrix $C_{(i k)(j l)}=B_{i k}^{*} A_{i j} B_{j l}$ (where the pairs $(i k)$ and $(j l)$ are considered as a single index) is in $Q_{m}$. Since $\phi$ is completely strongly positive, we have from the above equation

$$
\sum_{i j=1}^{n}\left(F_{i}, \pi\left(A_{i j}\right) F_{j}\right) \geq 0 .
$$

Since vectors of the form (a) for $r=1,2, \ldots$ exhaust $D$, it follows that the above equation is valid for all $F_{i} \in \mathcal{D}$. Now suppose $F_{i} \in \mathscr{D}(\pi)$ for $i=1, \ldots, n$. Since $\mathscr{D}$ is strongly dense in $\mathscr{D}(\pi)$, there are sequences $\left\{F_{i k} \in \mathcal{D} ; i=1, \ldots, n, k=1,2, \ldots\right\}$ such that

$$
\left\|F_{j k}-F_{j}\right\|+\sum_{i=1}^{n}\left\|\pi\left(A_{i j}\right)\left(F_{j k}-F_{j}\right)\right\| \rightarrow 0
$$


as $k \rightarrow \infty$. Hence, we have

$$
\sum_{i j=1}^{n}\left(F_{i}, \pi\left(A_{i j}\right) F_{j}\right)=\lim _{k \rightarrow \infty} \sum_{i j=1}^{n}\left(F_{i k}, \pi\left(A_{i j}\right) F_{j k}\right) \geq 0
$$

for all $F_{i} \in \mathscr{D}(\pi), i=1, \ldots, n$. Since $\left\{A_{i j}\right\}$ is an arbitrary element of $Q_{m}$, we have shown that $\pi$ is completely strongly positive.

Finally, we show that the representation $\pi$ is determined up to unitary equivalence. Suppose that $\pi^{\prime}$ is a closed *-representation of $\mathfrak{A}$ on a Hilbert space $\mathfrak{H}^{\prime}$ and $V^{\prime}$ is a linear mapping of $\mathcal{K}$ into $\mathcal{D}\left(\pi^{\prime}\right)$ such that $\langle f|\phi(A)| g\rangle=\left(V^{\prime} f, \pi^{\prime}(A) V^{\prime} g\right)$ for all $f, g \in \mathcal{K}$ and $A \in \mathbb{Q}$. Suppose further that $\left\{\pi^{\prime}(\mathfrak{Q}) V \mathcal{K}\right\}$ is strongly dense in $\mathcal{D}\left(\pi^{\prime}\right)$. We show that $\pi$ and $\pi^{\prime}$ are unitarily equivalent.

We begin by defining a linear mapping $U$ from $\mathscr{D} \subset \mathscr{D}(\pi)$ to $\left\{\pi^{\prime}(\mathscr{Q}) V^{\prime} \mathcal{K}\right\}$ by the relation

We have

$$
U \sum_{i=1}^{n} \pi\left(A_{i}\right) V f_{i}=\sum_{i=1}^{n} \pi^{\prime}\left(A_{i}\right) V^{\prime} f_{i}
$$

$$
\begin{aligned}
\left\|U \sum_{i=1}^{n} \pi\left(A_{i}\right) V f_{i}\right\|^{2} & =\left\|\sum_{i=1}^{n} \pi^{\prime}\left(A_{i}\right) V^{\prime} f_{i}\right\|^{2} \\
& =\sum_{i j=1}^{n}\left(\pi^{\prime}\left(A_{i}\right) V^{\prime} f_{i}, \pi^{\prime}\left(A_{j}\right) V^{\prime} f_{j}\right)=\sum_{i j=1}^{n}\left\langle f_{i}\left|\phi\left(A_{i}^{*} A A_{j}\right)\right| f_{j}\right\rangle \\
& =\sum_{i j=1}^{n}\left(\pi\left(A_{i}\right) V f_{i}, \pi\left(A_{j}\right) V f_{j}\right)=\left\|\sum_{i=1}^{n} \pi\left(A_{i}\right) V f_{i}\right\|^{2} .
\end{aligned}
$$

Hence, $U$ is well defined and isometric. Since $U$ maps a dense set in $\mathcal{H}$ onto a dense set in $\mathcal{H}^{\prime}, U$ has a unique extension to an isometry of $\mathcal{H}$ onto $\mathfrak{H}^{\prime}$. We also denote this extension by $U$. A straightforward computation (like the one which showed $U$ was isometric) shows $U$ is bicontinuous from $\mathscr{D}$ to $\left\{\pi^{\prime}(\mathfrak{Q}) V^{\prime} \mathcal{K}\right\}$ in the induced topologies of $\mathscr{D}$ and $\left\{\pi^{\prime}(\mathscr{Q}) V^{\prime} \mathcal{K}\right\}$. Since $\mathscr{D}(\pi)$ and $\mathscr{D}\left(\pi^{\prime}\right)$ are the completions of $\mathscr{D}$ and $\left\{\pi^{\prime}(\mathscr{Q}) V^{\prime} \mathcal{K}\right\}$ in the induced topologies, it follows that $U$ maps $\mathscr{D}(\pi)$ onto $\mathscr{D}\left(\pi^{\prime}\right)$. From the construction of $U$ we have $U \pi(A) f=\pi^{\prime}(A) U f$ for all $A \in \mathbb{Q}$ and $f \in \mathscr{D}(\pi)$. Hence, $\pi$ and $\pi^{\prime}$ are unitarily equivalent. This completes the proof of the theorem.

III. A generalization of Arveson's extension theorem. In this section we prove a generalization of a theorem of Arveson concerning the extension of completely positive maps. In [1] Arveson proves that if $S$ is a normed closed selfadjoint linear subspace of a $C^{*}$-algebra $\mathfrak{Q}$ with unit and the unit $I \in S$ and $\phi$ is a completely positive map defined on $S$ then $\phi$ has a completely positive extension to all of $\mathfrak{A}$. Arveson remarks that the requirement $I \in S$ can be weakened. 
We wish to prove Arveson's theorem in a form which can be applied to algebras of unbounded operators. In this case the unit $I$ is no longer an interior point of the cone of positive elements and the topological structure associated with a $C^{*}$ algebra is no longer available. For this reason we state and prove Arveson's theorem in a more abstract form which is independent of topology and algebraic structure.

Suppose $X$ is a complex linear vector space with a conjugate linear involution $x \rightarrow x^{*}$ with the properties

(i) $(\alpha x+y)^{*}=\bar{\alpha} x^{*}+y^{*}$,

(ii) $x^{* *}=x$,

for all $x, y \in X$ and complex numbers $a$. We denote by $M(X)$ the space of all finite matrices over $X$. Each element $\left\{x_{i j}\right\} \in M(X)$ is an array $x_{i j} \in X$ for $i, j=$ $1,2, \ldots$ with $x_{i j} \neq 0$ for only finitely many pairs of indices. We define a *operation on $M(X)$ by the relation

$$
\left\{x_{i j}\right\}^{*}=\left\{y_{i j}\right\} \text { if } y_{i j}=x_{j i}^{*}
$$

for all $i, j=1,2, \ldots$ We denote by $M(X)$, the hermitian elements of $M(X)$, those elements such that $\left\{x_{i j}\right\}=\left\{x_{i j}\right\}^{*}$.

Definition 3.1. We say a cone $Q_{m}$ in $M(X){ }_{r}$ is admissible if

(i) $Q_{m}$ is a cone in that if $\left\{x_{i j}\right\},\left\{y_{i j}\right\} \in Q_{m}$ and $\lambda, \mu \geq 0$ then $\lambda\left\{x_{i j}\right\}+\mu\left\{y_{i j}\right\} \in Q_{m}$.

(ii) If $\left\{x_{i j}\right\} \in Q_{m}$ and $\left\{a_{i j} ; i, j=1,2, \ldots\right\}$ is an array of complex numbers such that $a_{i j} \neq 0$ for only finitely many pairs $(i, j)$ of indices and $y_{i j}=$ $\sum_{k, l=1}^{\infty} \bar{a}_{k i} a_{l j} x_{k l}$ then $\left\{y_{i j}\right\} \in Q_{m}$.

The definition of an admissible cone is the same as the definition of an algebraically admissible cone, as given in the last section, except that here we only consider multiplication by scalars. It is clear that if $X$ is a ${ }^{*}$-algebra then an algebraically admissible cone is also an admissible cone.

As in the last section, if $\mathcal{K}$ is a complex vector space we denote by $\mathbb{B}(\mathcal{K})$ the space of (conjugate linear-linear) bilinear forms on $\mathcal{K}$.

Definition 3.2. Suppose $Q_{m}$ is an admissible cone in $M(X)_{r}$ and $K$ is a complex vector space. A completely positive (with respect to $Q_{m}$ ) map $\phi$ of $X$ on $K$ is a linear map $\phi$ of $X$ into $B(K)$ such that if $\left\{x_{i j}\right\} \in Q_{m}$ then

$$
\sum_{i j=1}^{\infty}\left\langle f_{i}\left|\phi\left(x_{i j}\right)\right| f_{j}\right\rangle \geq 0
$$

for all $f_{i} \in \mathcal{K}, i=1,2, \ldots$.

If $Q_{m}$ is an admissible cone in $M(X)_{r}$, we say an element $x=x^{*} \in X$ is in $Q_{m}\left(x \in Q_{m}\right)$ if the matrix $\left\{x \delta_{1 i} \delta_{1 j}\right\} \in Q_{m}$.

Definition 3.3. A symmetric (i.e. $x \in \Re$ implies $x^{*} \in \mathbb{M}$ ) linear subspace $\Re$ of $X$ is said to be cofinal in $X$ (with respect to an admissible cone $Q_{m}$ in $M(X)$ ) 
if for every $x=x^{*} \in X$ there is a $y=y^{*} \in \mathbb{M}$ with $y \in Q_{m}$ and $y-x \in Q_{m}$.

We can now state the main result of this section. We will show that if $M$ is a symmetric cofinal subspace of $X$ with respect to an admissible cone $Q_{m}$ in $M(X)_{r}$ and $\phi$ is a completely positive map of $\mathbb{M}$ on $\mathcal{K}$ then $\phi$ has a completely positive extension to $X$ as a completely positive map on $K$. This result is a generalization of Arveson's theorem on extensions of completely positive maps on $C^{*}$-algebras.

Lemma 3.4. Suppose $M$ is cofinal in $X$ with respect to an admissible cone $Q_{m}$ in $M(X)_{r}$. Then for every $\left\{x_{i j}\right\} \in M(X)_{r}$, there is an element $\left\{y_{i j}\right\} \in M(\mathbb{M})$, (i.e., $y_{i j} \in \mathbb{M}$ for $\left.i, j=1,2, \ldots\right)$ with $\left\{y_{i j}\right\} \in Q_{m}$ and $\left\{y_{i j}\right\}-\left\{x_{i j}\right\} \in Q_{m^{*}}$

Proof. The proof of this lemma is based on the fact that for each element $\left\{x_{i j}\right\} \in M(X)$, there are an integer $n$ and complex numbers $\left\{a_{i k} ; i=1,2, \ldots, k=\right.$ $1, \ldots, n\}\left(\alpha_{i k} \neq 0\right.$ for only finitely many integers $\left.i\right)$ and $x_{k}=x_{k}^{*} \in X$ for $k=1$, $\cdots, n$ so that

$$
x_{i j}=\sum_{k=1}^{n} \bar{\alpha}_{i k} x_{k} \alpha_{j k} .
$$

We leave the proof of this result to the reader with the remark that once one has proved the result for $(2 \times 2)$-matrices in $M(X)$, the general result follows from the fact that each $\left\{x_{i j}\right\} \in M(X)_{r}$ can be expressed as a sum of rank 2 matrices in $M(X)_{r}$.

Suppose $M$ is cofinal in $X$ and $\left\{x_{i j}\right\} \in M(X)_{r}$; then the $x_{i j}$ can be expressed in the form (a) above. Since $\mathbb{M}$ is cofinal in $X$, there are $y_{k}=y_{k}^{*} \in \mathbb{M}$ with $y_{k} \in Q_{m}$ and $y_{k}-x_{k} \in Q_{m}$ for $k=1, \ldots, n$. Let $y_{i j}=\sum_{i j=1}^{n} \bar{a}_{i k} a_{j k} y_{k}$; then $\left\{y_{i j}\right\} \in Q_{m}$ and $\left\{y_{i j}\right\}-\left\{x_{i j}\right\} \in Q_{m}$ from the properties of admissible cones. This completes the proof of the lemma.

Lemma 3.5. Suppose $X$ is a real linear vector space and $\left\{x_{a} \in X, a_{a} \in \mathbf{R}\right.$; $a \in I\}$ is a set of pairs of elements $x_{a} \in X$ and real numbers $a_{a}$ indexed by the set 1 . Let

$$
\begin{gathered}
S_{1}=\left\{x \in X ; x=-\sum_{i=1}^{n} \lambda_{i} x_{i}, \lambda_{i} \geq 0, i=1, \ldots, n\right. \\
\text { and } \left.\sum_{i=1}^{n} \lambda_{i} a_{a_{i}}<1, n=1,2, \ldots\right\}, \\
V_{1}=\left\{x \in X ; x=\sum_{i=1}^{n} \lambda_{i} x_{i}, \lambda_{i} \geq 0, i=1, \ldots, n\right\} \\
\text { and } \left.\sum_{i=1}^{n} \lambda_{i} a_{a_{i}} \leq-1, n=1,2, \ldots\right\} .
\end{gathered}
$$


If 0 is an internal point of $S_{1}$ then one and only one of the following statements is true:

(a) There exists a real linear functional $F$ on $X$ such that $F\left(x_{a}\right)+a_{\alpha} \geq 0$ for all $a \in I$.

(b) $0 \in V_{1}$.

Proof. If $V_{1}$ is empty then $F(x)=0$ satisfies statement (a) and statement (b) is false. We will assume hereafter that $V_{1}$ is not empty.

Assume that both (a) and (b) are true. Then from statement (b) we have $0=$ $\sum_{i=1}^{n} \lambda_{i} x_{a_{i}}$ with $\lambda_{i} \geq 0$ for $i=1, \ldots, n$ and $\sum_{i=1}^{n} \lambda_{i} a_{a_{i}} \leq-1$. If $F$ is a linear functional satisfying (a) then $F\left(x_{a_{i}}\right)+a_{a_{i}} \geq 0$ for $i=1, \ldots, n$. Hence, we have

$$
\sum_{i=1}^{n} \lambda_{i}\left(F\left(x_{a_{i}}\right)+a_{a_{i}}\right) \geq 0, \quad F\left(\sum_{i=1}^{n} \lambda_{i} x_{a_{i}}\right) \geq-\sum_{i=1}^{n} \lambda_{i} a_{a_{i}} \geq 1 .
$$

Hence, $F(0) \geq 1$ which is a contradiction. Therefore, statements (a) and (b) cannot both be true.

Next suppose 0 is an internal point of $S_{1}$ and $S_{1}$ and $V_{1}$ are disjoint. Since $S_{1}$ and $V_{1}$ are convex sets one of which has an internal point, the basic separation theorem for convex sets (see [3, Theorem 12, Chapter V, p. 412]) assures us that there exist a nonzero linear functional $f$ on $X$ and a real number $c$ such that $f(x) \geq c$ for all $x \in V_{1}$ and $f(x)<c$ for all $x \in S_{1}$. Since 0 is an internal point of $S_{1}$ and $f$ is nonzero, it follows that $c>0$. Let $F(x)=c^{-1} f(x)$ for all $x \in X$. We claim $F$ satisfies statement (a). To this end suppose $a_{a} \geq 0$. Then $-\left(a_{a}+\epsilon\right)^{-1} x_{a}$ $\epsilon S_{1}$ for all $\epsilon>0$. Then $F\left(-\left(a_{a}+\epsilon\right)^{-1} x_{a}\right) \leq 1$ which implies $F\left(x_{a}\right)+a_{a}+\epsilon \geq 0$ for all $\epsilon>0$. Hence, $F\left(x_{a}\right)+a_{a} \geq 0$ for all $a \in I$ with $a_{a} \geq 0$. Next suppose $a_{a} \geq 0$. Then $-a_{a}^{-1} x_{a} \in V_{1}$ and, therefore, $F\left(-a_{\alpha}^{-1} x_{\alpha}\right) \geq 1$ which implies $F\left(x_{\alpha}\right)+a_{a} \geq 0$. Hence, $F\left(x_{a}\right)+a_{a} \geq 0$ for all $a \in I$. Hence, we have shown that if $S_{1}$ and $V_{1}$ are disjoint, statement (a) is true. Hence, if statement (a) is false $S_{1}$ and $V_{1}$ are not disjoint and there is an $x \in X$ which lies in both $S_{1}$ and $V_{1}$, i.e.,

$$
x=-\sum_{i=1}^{n} \lambda_{i} x_{a_{i}^{\prime}} \quad x=\sum_{j=1}^{m} \lambda_{j}^{\prime} x_{\beta_{j}},
$$

with $\lambda_{i}, \lambda_{j}^{\prime} \geq 0$ for $i=1, \ldots, n, j=1, \ldots, m$ and

$$
\sum_{i=1}^{n} \lambda_{i} a_{a_{i}}=\delta_{1}<1, \quad \sum_{j=1}^{m} \lambda_{j}^{\prime} a_{\beta_{j}}=\delta_{2} \leq-1 .
$$

We have $\delta=-\left(\delta_{1}+\delta_{2}\right)>0$. Then we have

$$
0=\delta^{-1} \sum_{i=1}^{n} \lambda_{i} x_{a_{i}}+\delta^{-1} \sum_{j=1}^{m} \lambda_{j}^{\prime} x_{\beta_{j}}
$$

and $\delta^{-1} \lambda_{i} \geq 0, \delta^{-1} \lambda_{j}^{\prime} \geq 0$ for $i=1, \cdots, n$ and $j=1, \cdots, m$ and 


$$
\sum_{i=1}^{n} \delta^{-1} \lambda_{i} a_{i}+\sum_{j=1}^{m} \delta^{-1} \lambda_{j}^{\prime} a_{\beta_{j}}=\delta^{-1}\left(\delta_{1}+\delta_{2}\right)=-1 \leq-1 .
$$

Hence, $0 \in V_{1}$. Hence if statement (a) is false statement (b) is true, and this completes the proof of the lemma.

Lemma 3.6. Suppose $\mathbb{M}$ is a symmetric linear subspace of $X$ which is cofinal in $X$ with respect to an admissible cone $Q_{m}$ in $M(X)$, and $\phi$ is a completely positive map of $M$ on $K$. Suppose $x_{0}=x_{0}^{*} \in X$ and $x_{0} \notin \Re$. Let $\Re^{\prime}$ be the span of $\mathbb{M}$ and $x_{0}$. Then there exists a completely positive map $\phi^{\prime}$ of $\mathbb{M}^{\prime}$ on $\mathcal{K}$ which extends $\phi$.

Proof. Suppose the hypotheses of the lemma are satisfied. In order to specify an extension $\phi^{\prime}$ of $\phi$ it is sufficient to specify $\phi^{\prime}\left(x_{0}\right)$. A completely positive extension $\phi^{\prime}$ exists if and only if there is a bilinear form $\phi^{\prime}\left(x_{0}\right)$ such that

$$
\sum_{i j=1}^{\infty} a_{i j}\left\langle f_{i}\left|\phi^{\prime}\left(x_{0}\right)\right| f_{j}\right\rangle+\left\langle f_{i}\left|\phi\left(x_{i j}\right)\right| f_{j}\right\rangle \geq 0
$$

for all $f_{i} \in \mathcal{K}$, complex numbers $a_{i j}=\bar{\alpha}_{j i}, x_{i j}=x_{j i}^{*} \in \mathbb{M}$ for $i, j=1,2, \ldots$ such that $\left\{a_{i j} x_{0}+x_{i j}\right\} \in Q_{m}$. This condition is similar to statement (a) of the previous lemma.

Let $\overline{\mathcal{K}} \otimes \mathcal{K}$ be the linear space of all expressions $y=\sum_{i=1}^{n} \bar{f}_{i} \otimes g_{i}$ with $f_{i}, g_{i} \in \mathcal{K}$ for $i=1, \ldots, n$ and $n=1,2, \ldots$ On $\bar{K} \otimes \mathcal{K}$ we have the relations

$$
\begin{aligned}
& \left(a f+f^{\prime}\right) \otimes g=\bar{a}(\bar{f} \otimes g)+\bar{f}^{\prime} \otimes g, \\
& \bar{f} \otimes\left(a g+g^{\prime}=\alpha(\bar{f} \otimes g)+\bar{f} \otimes g^{\prime},\right.
\end{aligned}
$$

We define a ${ }^{*}$-operation on $\mathcal{K}$ by the relation

if $y=\sum_{i=1}^{n} \bar{f}_{i} \otimes g_{i}$ then $y^{*}=\sum_{i=1}^{n} \bar{g}_{i} \otimes f_{i}$.

Let $Y$ be the real linear space of all $y \in \bar{K} \otimes \mathcal{K}$ such that $y=y^{*}$. We assert that each element $y \in Y$ can be expressed in the form $y=\sum_{i j=1}^{n} a_{i j}\left(\bar{f}_{i} \otimes f_{j}\right)$ where $a_{i j}=\bar{\alpha}_{j i}$. Furthermore, the element $y$ in the above form is equal to zero $(y=0)$ if and only if theie are elements $\left\{g_{i} \in \mathcal{K} ; i=1, \ldots, r\right\}$ with $r \leq n$ and a matrix $\left\{\gamma_{i j} ; i=1, \ldots, n\right.$ and $\left.j=1, \ldots, r\right\}$ so that $\Sigma_{j=1}^{r} \gamma_{i j} g_{j}=f_{i}$ and $\gamma^{*} a \gamma=0$, i.e.,

$$
\sum_{k, l=1}^{n} \bar{\gamma}_{k i} a_{k l} \gamma_{l j}=0 \text { for all } i, j=1, \ldots, r \text {. }
$$

Each element $C \in B(\mathcal{K})$ defines a linear functional $F_{C}$ on $\bar{K} \otimes K$ by the relation

$$
F_{C}(y)=\sum_{i=1}^{n}\left\langle f_{i}|C| g_{i}\right\rangle
$$


where $y=\sum_{i=1}^{n} \bar{f}_{i} \otimes g_{i}$.

If $C^{*}$ is the hermitian adjoint of $C \in \mathbb{B}(\mathcal{K})$ (i.e., $\left\langle f\left|C^{*}\right| g\right\rangle=\langle\overline{g|C| f}\rangle$ for all $f, g \in \mathcal{K})$ we have $F_{C *}(y)=\overline{F_{C}\left(y^{*}\right)}$. It follows that each hermitian $C \in B(K)$ (i.e. $C=C^{*}$ ) defines a real linear functional on $Y$. In fact there is a one-to-one correspondence between real linear functionals on $Y$ and hermitian forms in $B(K)$.

In terms of the real linear space $Y$ the question of whether there is a hermitian form $\phi^{\prime}\left(x_{0}\right)$ satisfying condition $\left(a^{\prime}\right)$ is equivalent to the question of whether there exists a real linear functional $F$ on $Y$ such that

$$
F\left(y_{a}\right)+a_{a} \geq 0
$$

for all $y_{a}=\sum_{i j=1}^{n} a_{i j}\left(\bar{f}_{i} \otimes f_{j}\right) \in Y, a_{\alpha}=\sum_{i j=1}^{n}\left\langle f_{i}\left|\phi\left(x_{i j}\right)\right| f_{j}\right\rangle$ where $\left\{a_{i j} x_{0}+x_{i j}\right\} \in Q_{M}$ and $n=1,2, \ldots$. Let $S_{1}$ and $V_{1}$ be as in Lemma 3.5. We show 0 is an internal point of $S_{1}$. Suppose $y=\sum_{i j=1}^{n} a_{i j}\left(\bar{f}_{i} \otimes f_{j}\right)$. Since $\mathbb{\eta}$ is cofinal in $X$, it follows from Lemma 3.4 that there is an $\left\{x_{i j}\right\} \in M(M)$, such that $\left\{x_{i j}\right\} \in Q_{m}$ and $\left\{x_{i j}-x_{0} \alpha_{i j}\right\} \in Q_{m}$. Let $a=\sum_{i j=1}^{n}\left\langle f_{i}\left|\phi\left(x_{i j}\right)\right| f_{j}\right\rangle \geq 0$. Then if $F$ is to satisfy condition (a") above we have $F(-y)+a \geq 0$. Suppose $0 \leq \lambda<(a+1)^{-1}$. We have $\lambda a<a(a+1)^{-1}$. Then from the definition of $S_{1}$ we have $\lambda y \in S_{1}$ since $\lambda a<1$. Hence, 0 is an internal point of $S_{1}$.

Then it follows from Lemma 3.5 that either ( $\left(a^{\prime \prime}\right)$ has a solution or $0 \in V_{1}$. Suppose inequality $\left(a^{\prime \prime}\right)$ has no solution and, therefore, $0 \in V_{1}$. Since $0 \in V_{1}$ there are $y_{k}=\Sigma_{i j=1}^{m(k)} a(k)_{i j} \bar{f}_{i k} \otimes f_{j k}, k=1, \cdots, n,\left\{x_{i j}^{(k)}\right\} \in M(\mathbb{D})$, with $\left\{a(k)_{i j} x_{0}+x_{i j}^{(k)}\right\} \in Q_{m}$ and numbers $\lambda_{k} \geq 0$ for $k=1, \ldots, n$ so that

$$
\sum_{k=1}^{n} \lambda_{k} y_{k}=0 \text { and } \sum_{k=1}^{n} \sum_{i j=1}^{m(k)} \lambda_{k}\left\langle f_{i k}\left|\phi\left(x_{i j}^{(k)}\right)\right| f_{j k}\right\rangle \leq-1 \text {. }
$$

Let $a_{(i k)(j l)}=\lambda_{k} \delta_{k l} a(k)_{i j}$ and $x_{(i k)(j l)}=\lambda_{k} \delta_{k l} x_{i j}^{(k)}$. Then combining the pair of indices ( $i k)$ into a single index $i$ and the pair of indices $(j l)$ into a single index $j$ (where now $i, j=1, \ldots, m$ ) we have

$$
y=\sum_{i j=1}^{m} a_{i j} \bar{f}_{i} \otimes f_{j}=\sum_{i j k l} a_{(i k)(j l)} \bar{f}_{i k} \otimes f_{j l}=0,
$$

$\left\{a_{i j} x_{0}+x_{i j}\right\} \in Q_{m}$ with $x_{i j}=x_{j i}^{*} \in \mathbb{M}$ for $i, j=1, \ldots, m$, and

$$
\sum_{i j=1}^{m}\left\langle f_{i}\left|\phi\left(x_{i j}\right)\right| f_{j}\right\rangle \leq-1 \text {. }
$$

Since $y=0$, there are $g_{i} \in \mathcal{K}$ for $i=1, \ldots, r$ (with $r \leq m$ ) and a matrix $\left\{\gamma_{i j} ; i=\right.$ $1, \ldots, m, y=1, \ldots, r\}$ such that

$$
f_{i}=\sum_{j=1}^{r} \gamma_{i j} g_{j} \text { for } i=1, \cdots, m
$$


and $\left(\gamma^{*} a \gamma\right)_{i j}=\Sigma_{k, l=1}^{m} \bar{\gamma}_{k i} a_{k l} \gamma_{k j}=0$ for all $i, j=1, \ldots, m$. Now from the properties of an admissible cone we have

$$
\left\{\gamma ^ { * } \left\{\left\{a_{i j} x_{0}+x_{i j}\right\}\{\gamma\}=\left\{z_{i j}\right\} \in Q_{m}\right.\right.
$$

with $z_{i j}=\sum_{k l=1}^{m} \bar{\gamma}_{k i} x_{k l} \gamma_{l j}$. Note the $\left\{\alpha_{i j} x_{0}\right\}$ terms drop out since $\gamma^{*} a \gamma=0$. We have

$$
\sum_{i j=1}^{r}\left\langle g_{i}\left|\phi\left(z_{i j}\right)\right| g_{j}\right\rangle=\sum_{i k j l}\left\langle\gamma_{i j} g_{k}\left|\phi\left(x_{i j}\right)\right| \gamma_{i l} g_{l}\right\rangle=\sum_{i j=1}^{m}\left\langle f_{i}\left|\phi\left(x_{i j}\right)\right| f_{j}\right\rangle \leq-1 .
$$

But since $\left\{z_{i j}\right\} \in Q_{m}$ this contradicts the complete positivity of $\phi$ on $\Re$ and we have reached a contradiction. Hence, inequality $\left(a^{\prime \prime}\right)$ has a solution. Hence, inequality $\left(a^{\prime}\right)$ has a solution and, therefore, $\phi$ has a completely positive extension to $\mathrm{M}^{\prime}$. This completes the proof of the lemma.

Theorem 3.7. Suppose $M$ is a symmetric linear subspace of $X$ which is cofinal in $X$ with respect to an admissible cone $Q_{m}$ in $M(X)_{r}$ and $\phi$ is a completely positive map of $\Re$ on $\mathcal{K}$. Then $\phi$ bas a completely positive extension to $X$, i.e. there exists a completely positive map $\phi^{\prime}$ of $X$ on $\mathcal{K}$ such that $\phi^{\prime} \mid m=\phi$.

Proof. The proof follows immediately from an application of Zorn's lemma to the preceding lemma.

IV. Standard representations of enveloping algebras of Lie algebras. In [4] Nelson showed that there are selfadjoint representations of enveloping algebras of Lie algebras which cannot be exponentiated to give a unitary representation of the Lie group. In this same paper Nelson showed that a representation $\pi$ of the enveloping algebra of a Lie algebra can be exponentiated to give a unitary representation of the Lie group if a certain second order elliptic element $A$ of the enveloping algebra is represented by an operator $\pi(A)$ which has a dense set of analytic vectors. In this section we show that a selfadjoint representation of the enveloping algebra of a Lie algebra can be exponentiated to give a strongly continuous unitary representation of the Lie group if and only if the representation is completely strongly positive with respect to an admissible cone $Q_{m}$ which will be defined shortly.

Throughout this section we will let $G$ denote an arbitrary connected simply connected Lie group and $\mathscr{L}(G)$ will denote its Lie algebra. We will let $\mathscr{Q}(G)$ denote the universal enveloping algebra of $\mathcal{L}(G)$. Specifically if $\left\{X_{1}, \ldots, X_{n}\right\}$ is a basis for $\mathscr{L}(G)$ then $\mathscr{Q}(G)$ consists of all noncommutative polynomials in the $X_{i}$ 's modulo the relations

$$
X_{i} X_{j}-X_{j} X_{i}=\sum_{k=1}^{n} C_{i j}^{k} X_{k}
$$


where the $\left\{C_{i j}^{k}\right\}$ are the structure constants of $\mathscr{L}(G)$ in this basis. We define a * operation on $\mathscr{L}(G)$ by specifying $X_{i}^{*}=-X_{i}$ for $X_{i} \in \mathscr{L}(G), i=1, \ldots, n$.

If $A \in \mathbb{Q}(G)$ is an element of the enveloping algebra we denote by $d(A)$ the right invariant differential operator on the group manifold associated with $A$. The mapping $A \rightarrow d(A)$ is a representation of $\mathbb{Q}(G)$ in that $d(\alpha A+B C)=\alpha d(A)+$ $d(B) d(C)$ for all $A, B, C \in \mathbb{Q}(G)$ and all complex numbers $a$. It follows that the mapping $A \rightarrow d(A)$ is completely specified once it has been specified for all $X \in \mathfrak{L}(G)$. Let $X \rightarrow g(X)=\exp (X)$ be the exponential map from $\mathcal{L}(G)$ into $G$. If $f$ is a $C^{\infty}$-function on the group $G$ we define $d(X)$ on $f$ by the relation

$$
(d(X) f)(g)=\lim _{t \rightarrow 0} \frac{f(g(t X) g)-f(g)}{t} .
$$

For each strongly continuous unitary representation $g \rightarrow U(g)$ of $G$ on a Hilbert space $\mathcal{H}$ there is an associated selfadjoint representation of $\mathscr{Q}(G)$ on $\mathcal{H}$ given as follows. Let $\mathcal{D}(\pi)$ be the linear manifold of all $C^{\infty}$-vectors for $U(g)$, i.e. $\mathscr{D}(\pi)$ consists of all $f \in \mathcal{H}$ such that the mapping $g \rightarrow U(g) f$ is an infinitely differentiable function of $G$ into $\mathcal{H}$. (We remark that it is enough to know that $(f, U(g) f)$ is a $C^{\infty}$-function on $G$.) The $C^{\infty}$-vectors $\mathscr{D}(\pi)$ form a dense linear manifold in $\mathcal{H}$, since $\mathcal{D}(\pi)$ contains the Garding domain which is well known to be dense in $\mathcal{H}$. The representation $\pi$ associated with the representation $g \rightarrow U(g)$ is given by

$$
\pi(A) f=\left.d(A) U(g) f\right|_{g=e}
$$

for all $A \in \mathbb{Q}(G)$ and $f \in \mathscr{D}(\pi)$ and $e$ denotes the unit element of $G$.

One can easily verify that $\pi$ is a ${ }^{*}$-representation of $\mathscr{Q}(G)$ on $\mathcal{H}$. We show $\pi$ is selfadjoint. Suppose $f \in \mathscr{D}\left(\pi^{*}\right)$. We will show that the function $F(g)=(f, U(g) f)$ is a $C^{\infty}$-function on $G$ and, thus, $f \in \mathcal{D}(\pi)$. Since $\mathscr{D}(\pi)$ is dense in $\mathcal{H}$, there is a sequence $b_{n} \in \mathcal{D}(\pi)$ such that $\left\|b_{n}-f\right\| \rightarrow 0$ as $n \rightarrow \infty$. Let $F_{n}(g)=\left(f, U(g) b_{n}\right)$. Since $U(g) \mathscr{D}(\pi) \subset \mathscr{D}(\pi)$ for all $g \in G$ and $f \in \mathscr{D}\left(\pi^{*}\right)$ we have

$$
\left(d(A) F_{n}\right)(g)=\left(f, \pi(A) U(g) b_{n}\right)=\left(\pi^{*}\left(A^{*}\right) f, U(g) b_{n}\right) .
$$

We then have that $F(g)=(f, U(g) f)$ is the uniform limit of a sequence of $C^{\infty}$-functions and, furthermore, each differential operator $d(A)$ with $A \in \mathbb{Q}(G)$ when applied to this sequence produces a uniformly convergent sequence of functions. Hence, $F$ is a $C^{\infty}$-function on $G$ and, in fact,

$$
(d(A) F)(g)=\left(\pi^{*}\left(A^{*}\right) f, U(g) f\right)
$$

for all $g \in G$ and $A \in \mathbb{Q}(G)$. Hence, $f$ is a $C^{\infty}$-vector for $U(g)$ and, thus, $f \in \mathscr{D}(\pi)$. Therefore, we have $\mathscr{D}(\pi) \supset \mathscr{D}\left(\pi^{*}\right)$ and, hence, $\mathscr{D}(\pi)=\mathscr{D}\left(\pi^{*}\right)$ and $\pi$ is selfadjoint.

Definition 4.1. A *-representation $\pi$ of $\mathscr{Q}(G)$ on a Hilbert space $\mathcal{H}$ is said to be standard if $\pi$ is selfadjoint and $\pi$ is associated with some strongly continuous unitary representation of $G$ on $\mathcal{H}$ in the manner described. 
Speaking more informally a standard representation of $\mathbb{Q}(G)$ is a selfadjoint representation in which the operators $-i \pi\left(X_{j}\right)$ are essentially selfadjoint for $j=1, \ldots, n$ and can be exponentiated to give a strongly continuous unitary representation of $G$ on $\mathcal{H}$.

Lemma 4.2. Suppose $\pi$ is a standard representation of $\mathbb{Q}(G)$ on a Hilbert space $\mathcal{H}$ and $\pi_{1}$ is a selfadjoint subrepresentation of $\pi$. Then $\pi_{1}$ is standard.

Proof. Suppose $\pi$ and $\pi_{1}$ satisfy the hypotheses of the lemma. Since $\pi_{1}$ is a selfadjoint subrepresentation of $\pi$, it follows from Theorem 1.12 that there is a hermitian projection $E \in \pi(\mathfrak{Q})^{\prime}$ such that $\mathscr{D}\left(\pi_{1}\right)=E \mathscr{D}(\pi)$. Since $\pi$ is standard, we have $-i \overline{\pi(X)}$ is selfadjoint for all $X \in \mathscr{L}(G)$ and if $g \rightarrow U(g)$ is the strongly continuous unitary representation of $G$ to which $\pi$ is associated then $U(g(X))=$ $\exp (\overline{\pi(X)})$ for all $X \in \mathcal{L}(G)$ where $X \rightarrow g(X)$ is the exponential map from $\mathcal{L}(G)$ into $G$. It follows that $U(g(X))$ commutes with $E$ for all $X \in \mathscr{L}(G)$. Since each $g \in G$ can be expressed as a product of $g(X)$ 's, it follows that $U(g)$ commutes with $E$ for all $g \in G$. Let $g \rightarrow V(g)=U(g) \mid E \mathcal{H}$ be the restriction of the representation $g \rightarrow U(g)$ to the invariant subspace $\{E \mathcal{H}\}$. Now, we have $\mathscr{D}\left(\pi_{1}\right)=E \mathscr{D}(\pi)$ consists of the $C^{\infty}$-vectors for $V(g)$ and, for $f \in \mathscr{D}\left(\pi_{1}\right)$,

$$
\pi_{1}(A) f=\pi(A) f=\left.d(A) U(g) f\right|_{g=e}=\left.d(A) V(g) f\right|_{g=e} .
$$

Hence, $\pi_{1}$ is standard. This completes the proof of the lemma.

The main result of this section is that a selfadjoint representation of $\mathbb{Q}(G)$ on a Hilbert space $\mathcal{H}$ is standard if and only if it is completely strongly positive with respect to an algebraically admissible cone $Q_{m}$ in $M(\mathscr{Q}(G))$ which we will now define.

Definition 4.3. A complex $(n \times n)$-matrix valued function $E_{i j}(\cdot)$ defined on the group $G$ is said to be positive definite if

$$
\sum_{i j=1}^{n} \sum_{k l=1}^{m} \bar{a}_{i k} a_{j l} E_{i j}\left(g_{i k}^{-1} g_{j l}\right) \geq 0
$$

for all $g_{i k} \in G$ and complex numbers $a_{i k}$ for $i=1, \ldots, n$ and $j=1, \ldots, m$ where $m=1,2, \ldots$.

If $g \rightarrow U(g)$ is a unitary representation of $G$ on a Hilbert space $\mathcal{H}$ then the $(n \times n)$-matrix valued function $E_{i j}(g)=\left(f_{i}, U(g) f_{j}\right)$ with $f_{i} \in \mathcal{H}$ for $i=1, \ldots, n$ is positive definite since

$$
\sum_{i j=1}^{n} \sum_{k l=1}^{m} \bar{a}_{i k} \alpha_{j l}\left(f_{i}, U\left(g_{i k}^{-1} g_{j l}\right) f_{j}\right)=\sum_{i j=1}^{n} \sum_{k l=1}^{m}\left(a_{i k} U\left(g_{i k}\right) f_{i}, a_{j l} U\left(g_{j l}\right) f_{j}\right) \geq 0 .
$$

Conversely, Stinespring's construction for completely positive maps (discussed in \$II) shows that if $E_{i j}(\cdot)$ is a positive definite matrix valued function on $G$ then there is a unitary representation $g \rightarrow U(g)$ of $G$ on a Hilbert space $\mathcal{H}$ and vectors 
$f_{i} \in \mathcal{H}$ for $i=1, \ldots, n$ so that

$$
E_{i j}(g)=\left(f_{i}, U(g) f_{j}\right)
$$

Furthermore, if the matrix valued function $E_{i j}(\cdot)$ is continuous the representation $g \rightarrow U(g)$ may be chosen to be strongly continuous. Also, if the matrix valued function $E_{i j}(\cdot)$ is $C^{\infty}$ on $G$ the representation $g \rightarrow U(g)$ may be chosen to be strongly continuous and the vectors $f_{i} \in \mathcal{H}$ so that they are $C^{\infty}$-vectors for the representation.

We now define an algebraically admissible cone $Q_{m}$ in $M(\mathbb{Q}(G))$. Let $Q_{m}$ be the set of all $\left\{A_{i j}\right\}=\left\{A_{i j}\right\}^{*} \in M(\mathscr{Q}(G))$ such that

$$
\left.\sum_{i j=1}^{n} d\left(A_{i j}\right) E_{i j}(g)\right|_{g=e} \geq 0
$$

for all positive definite $(n \times n)$-matrix valued functions which are $C^{\infty}$ on $G$ for $n=1,2, \ldots$. One can easily verify that $\left\{A_{i j}\right\}=\left\{A_{i j}\right\} * Q_{m}$ if and only if

$$
\sum_{i j=1}^{\infty}\left(f_{i}, \pi\left(A_{i j}\right) f_{j}\right) \geq 0
$$

for all $f_{i} \in \mathscr{D}(\pi)$ for $i=1,2, \ldots$ for all standard representations $\pi$ of $\mathbb{Q}(G)$. From this remark it follows that $Q_{m}$ is an algebraically admissible cone in $M(\mathscr{Q}(G)$ ).

A *-representation $\pi$ of $\mathfrak{Q}(G)$ will be called completely strongly positive if it is completely strongly positive with respect to $Q_{m}$, i.e. if $\left\{A_{i j}\right\}=\left\{A_{i j}\right\}^{*} \in Q_{m}$ then

$$
\sum_{i j=1}^{\infty}\left(f_{i}, \pi\left(A_{i j}\right) f_{j}\right) \geq 0
$$

for all $f_{i} \in \mathscr{D}(\pi)$ for $i=1,2, \ldots$.

The main result of this section is that a selfadjoint representation of $\mathbb{Q}(G)$ on a Hilbert space is standard if and only if it is completely strongly positive. Before proving this result it will be useful to introduce what we shall call the universal standard representation of $\mathbb{Q}(G)$. This representation is constructed as follows.

Let $S_{n}$ be the set of all continuous positive definite $(n \times n)$-matrix valued functions on $G$. For each $E_{i j}(\cdot) \in S_{n}$ there is by the Stinespring construction discussed in \$II (see Theorem 2.4) a strongly continuous unitary representation $g \rightarrow V(g)$ on a Hilbert space $\mathcal{H}$ and vectors $f_{i} \in \mathcal{H}$ for $i=1, \ldots, n$ so that $E_{i j}(g)=\left(f_{i}, V(g) f_{j}\right)$ for $g \in G$ and $i, j=1, \ldots, n$ and the linear span of $\left\{V(g) f_{i}\right.$; $g \in G, i=1, \ldots, n\}$ is dense in $\mathcal{H}$. The representation $g \rightarrow V(g)$ is determined by this requirement up to unitary equivalence. In summary for each $E_{i j}(\cdot) \in S_{n}$ there is a strongly continuous unitary representation $g \rightarrow V(g)$ of $G$. Let $g \rightarrow$ $U^{(n)}(g)$ be the direct sum of all such representations $g \rightarrow V(g)$, one for each 
$E_{i j}(\cdot) \in S_{n}$. Finally, let $g \rightarrow U(g)$ be the direct sum of the representations $g \rightarrow$ $U^{(n)}(g)$ for $n=1,2, \ldots$ and let $\mathcal{K}$ be the Hilbert space on which $U(g)$ acts.

We call $g \rightarrow U(g)$ the universal strongly continuous unitary representation of $G$. This representation has the property that if $n$ is an arbitrary positive integer and $E_{i j}(\cdot)$ is a continuous positive definite $(n \times n)$-matrix valued function on $G$, then there are vectors $f_{i} \in \mathcal{K}$ for $i=1, \ldots, n$ so that $E_{i j}(g)=\left(f_{i}, U(g) f_{j}\right)$ for all $g \in G$ and $i, j=1, \cdots, n$.

Since $g \rightarrow U(g)$ is a strongly continuous unitary representation of $G$ on a Hilbert space $\mathcal{K}$, there is an associated selfadjoint representation $\pi_{u}$ of $\mathbb{Q}(G)$ on $\mathcal{K}$ which is constructed from the representation $g \rightarrow U(g)$ in the manner described at the beginning of this section. We will call $\pi_{u}$ the universal standard representation of $\mathbb{Q}(G)$. We note that an element $\left\{A_{i j}\right\}=\left\{A_{i j}\right\}^{*} \in M(\mathscr{Q}(G))$ is in $Q_{m}$ if and only if

$$
\sum_{i j=1}^{\infty}\left(f_{i}, \pi_{u}\left(A_{i j}\right) f_{j}\right) \geq 0
$$

for all $f_{i} \in \mathscr{D}\left(\pi_{u}\right)$ for $i=1,2, \ldots$. This follows from the definition of $Q_{m}$ and the universal property of the representation $g \rightarrow U(g)$.

Let $\mathfrak{Q}_{u}=\pi_{u}(\mathfrak{Q}(G))$ and let $\mathfrak{B}_{u}$ be the *-algebra generated by $\mathfrak{Q}_{u}$ and the unitary operators $U(g)$. This algebra will be of importance to us later in this section. We will construct $B_{u}$ more explicitly as follows. Each element $g \in G$ induces a linear transformation $T_{g}$ of the Lie algebra $\mathcal{L}(G) \subset \mathbb{Q}(G)$ by the relation

$$
U(g) \pi_{u}(X) U(g)^{-1}=\pi_{u}\left(T_{g}(X)\right)
$$

for all $X \in \mathfrak{L}(G) \subset \mathbb{Q}(G)$ and $g \in G$. It follows that each element $g$ induces a *automorphism $\alpha_{\boldsymbol{g}}$ of $\mathscr{Q}(G)$ by the relation

$$
U(g) \pi_{u}(A) U(g)^{-1}=\pi_{u}\left(a_{g}(A)\right)
$$

for all $A \in \mathbb{Q}(G)$ and $g \in G$. With the aid of this group of automorphisms $\left\{a_{g}\right\}$ we define the elements of $B_{u}$ and operations in $B_{u}$. Each element $A \in B_{u}$ can be expressed in the form $A=\sum_{i=1}^{n} \pi_{u}\left(A_{i}\right) U\left(g_{i}\right)$ with $A_{i} \in \mathbb{Q}(G)$ and $g_{i} \in G$ for $i=$ $1, \cdots, n$. If $B=\sum_{j=1}^{m} \pi_{u}\left(B_{j}\right) U\left(g_{j}^{\prime}\right)$ with $B_{j} \in \mathbb{Q}(G)$ and $g_{j} \in G$ for $j=1, \ldots, m$ then we have for the operations in $B_{u}$

$$
\begin{aligned}
A^{*} & =\sum_{i=1}^{n} U\left(g_{i}\right)^{*} \pi_{u}\left(A_{i}\right)^{*}=\sum_{i=1}^{n} U\left(g_{i}^{-1}\right) \pi_{u}\left(A_{i}^{*}\right) \\
& =\sum_{i=1}^{n} \pi_{u}\left(a_{B_{i}}{ }^{-1}\left(A_{i}^{*}\right)\right) U\left(g_{i}^{-1}\right)
\end{aligned}
$$


and

$$
A B=\sum_{i=1}^{n} \sum_{j=1}^{m} \pi_{u}\left(A_{i}\right) U\left(g_{i}\right) \pi_{u}\left(B_{j}\right) U\left(g_{j}^{\prime}\right)=\sum_{i=1}^{n} \sum_{j=1}^{m} \pi_{u}\left(A_{i} a_{g_{i}}\left(B_{j}\right)\right) U\left(g_{i} g_{j}^{\prime}\right) .
$$

We define $Q_{m}^{\prime}$ to be the natural algebraically admissible cone in $M\left(B_{u}\right)$. An element $\left\{A_{i j}\right\}=\left\{A_{i j}\right\}^{*} \in M\left(\mathscr{B}_{u}\right)$ is in $Q_{m}^{\prime}$ if and only if $\Sigma_{i j=1}^{\infty}\left(f_{i}, A_{i j} f_{j}\right) \geq 0$ for all $f_{i} \in \mathscr{D}\left(\pi_{u}\right)$ for $i=1,2, \ldots$. One easily checks that $Q_{m}^{\prime}$ is an algebraically admissible cone in $M\left(B_{u}\right)$. We take the order structure in $M\left(Q_{u}\right)$ to be the order structure inherited from $M\left(\mathbb{B}_{u}\right)$. We note that if $\left\{A_{i j}\right\}=\left\{A_{i j}\right\}^{*} \in M(\mathbb{Q}(G))$ then $\left\{A_{i j}\right\} \in Q_{m}$ if and only if $\left\{\pi_{u}\left(A_{i j}\right)\right\} \in Q_{m}^{\prime}$.

Next we note that $\mathfrak{C}_{u}$ is cofinal in $B_{u}$ with respect to the cone $Q_{m}^{\prime}$. To see that this is so, suppose $A=A^{*} \in B_{u}$. As we have seen, $A$ can be expressed in the form

$$
A=\sum_{i=1}^{m} \pi_{u}\left(A_{i}\right) U\left(g_{i}\right)
$$

with $A_{i} \in \mathbb{Q}(G)$ and $g_{i} \in G$ for $i=1, \ldots, m$. Since $A=A^{*}$, we have

$$
A=\sum_{i=1}^{m} U\left(g_{i}^{-1}\right) \pi_{u}\left(A_{i}^{*}\right) \text {. }
$$

Hence, we have

$$
A=\frac{1}{2} \sum_{i=1}^{m}\left(\pi_{u}\left(A_{i}\right) U\left(g_{i}\right)+U\left(g_{i}^{-1}\right) \pi_{u}\left(A_{i}^{*}\right)\right)
$$

Now, we have

$$
\begin{aligned}
& \left(\pi_{u}\left(A_{i}^{*}\right)-U\left(g_{i}\right)\right)^{*}\left(\pi_{u}\left(A_{i}^{*}\right)-U\left(g_{i}\right)\right) \\
& \quad=\pi_{u}\left(A_{i} A_{i}^{*}\right)-\pi_{u}\left(A_{i}\right) U\left(g_{i}\right)-U\left(g_{i}^{-1}\right) \pi_{u}\left(A_{i}^{*}\right)+I .
\end{aligned}
$$

Hence, we have $\pi_{u}\left(A_{i} A_{i}^{*}+I\right) \in Q_{m}^{\prime}$ and

$$
\pi_{u}\left(A_{i} A_{i}^{*}+I\right)-\pi_{u}\left(A_{i}\right) U\left(g_{i}\right)-U\left(g_{i}^{-1}\right) \pi_{u}\left(A_{i}^{*}\right) \in Q_{m}^{\prime} .
$$

Let $B=\sum_{i=1}^{m} \frac{1}{2 \pi} \pi\left(A_{i} A_{i}^{*}+I\right)$. We have $B \in \mathbb{Q}_{u} ; B \in Q_{m}^{\prime}$ and

$$
B-A=\frac{1}{2} \sum_{i=1}^{m}\left(\pi_{u}\left(A_{i} A_{i}^{*}+I\right)-\pi_{u}\left(A_{i}\right) U\left(g_{i}\right)-U\left(g_{i}^{-1}\right) \pi_{u}\left(A_{i}^{*}\right)\right) .
$$

Hence, $B-A \in Q_{m}^{\prime}$ and $\mathscr{Q}_{u}$ is cofinal in $B_{u}$.

We now prove a lemma which will be useful in the proof of the main theorem of this section. 
Lemma 4.4. Suppose $\pi$ is an order preserving *-representation of $B_{u}$ on a Hilbert space $\mathcal{H}$, where order preserving means $A \in B_{u}$ and $(f, A f) \geq 0$ for all $f \in \mathscr{D}\left(\pi_{u}\right)$ implies $(g, \pi(A) g) \geq 0$ for all $g \in \mathcal{D}(\pi)$. Let $\phi(A)=\pi\left(\pi_{u}(A)\right)$ for all $A \in \mathbb{Q}(G)$ and let $\mathscr{D}(\phi)=\mathscr{D}(\pi)$. Then $\phi$ bas a standard extension, i.e. there is a standard representation $\phi^{\prime}$ of $\mathfrak{Q}(G)$ on $\mathcal{H}$ such that $\phi^{\prime} \supset \phi$.

Proof. Suppose $\pi$ and $\phi$ satisfy the hypotheses of the lemma. We show $\phi$ has a standard extension.

Let $X \rightarrow g(X)=\exp (X)$ be the exponential map from the Lie algebra $\mathcal{L}(G)$ into the Lie group $G$. Let $X \in \mathscr{L}(G)$ and let $C(t)=U(g(t X))-I-t \pi_{u}(X)$ for all real $t$. We have $C(t) \in B_{u}$ and we will show $C(t)^{*} C(t) \leq 1 / 4 t^{4} \pi_{u}\left(X^{4}\right)$. Let $\{E(\lambda)$; $-\infty<\lambda<+\infty\}$ be the spectral resolution of the selfadjoint operator $-\mathrm{i} \pi(X)$ which is the generator of the one-parameter group $U(g(t X))=\exp \left(t \pi_{u}(X)\right)$. Then we have from the functional calculus for selfadjoint operators

$$
\|C(t) f\|^{2}=\left(f, C(t)^{*} C(t) f\right)=\int_{-\infty}^{+\infty}\left|e^{i t \lambda}-1-i t \lambda\right|^{2} d(f, E(\lambda) f)
$$

for all $f \in \mathscr{D}\left(\pi_{u}\right)$. Since $\left|e^{i t \lambda}-1-i t \lambda\right| \leq 1 / 2 \lambda^{2} t^{2}$ for all real $\lambda$ and $t$, we have

$$
\|C(t) f\|^{2} \leq \int_{-\infty}^{+\infty} 1 / 4 t^{4} \lambda^{4} d(f, E(\lambda) f)=1 / 4 t^{4}\left(f, \pi_{u}\left(X^{4}\right) f\right) .
$$

Hence, we have $C(t)^{*} C(t) \leq 1 / 4 t^{4} \pi_{u}\left(X^{4}\right)$ for all $X \in \mathfrak{L}(G)$ and all real $t$.

Since $\pi$ is an order preserving *-representation of $B_{u}$, we have

$$
\begin{aligned}
\|\pi(C(t)) f\|^{2} & =\left(f, \pi\left(C(t)^{*} C(t)\right) f\right) \leq 1 / 4 t^{4}\left(f, \pi\left(\pi_{u}\left(X^{4}\right) f\right)\right) \\
& =1 / 4 t^{4}\left(f, \phi\left(X^{4}\right) f\right)=\left(1 / 2 t^{2}\left\|\phi\left(X^{2}\right) f\right\|\right)^{2}
\end{aligned}
$$

for all $f \in \mathscr{D}(\pi)$. Let $V(g)=\pi(U(g))$. Since $\pi$ is a *-representation of $B_{u}$, we have $g \rightarrow V(g)$ is unitary representation of $G$. We have $\pi(C(t))=V(g(t X))-l-t \phi(X)$. Hence, we find from the above inequality after dividing by $t$ that

$$
\left\|t^{-1}(V(g(t X))-I) f-\phi(X) f\right\| \leq 1 / 2|t|\left\|\phi\left(X^{2}\right) f\right\|
$$

for all $X \in \mathfrak{L}(G), f \in \mathfrak{D}(\pi)$ and all real $t \neq 0$. It follows immediately from this estimate and the fact that $V(g) \mathscr{D}(\pi) \subset \mathscr{D}(\pi)$ for all $g \in G$, that the function $V(g) f$ is a $C^{1}$-vector function on $G$ and $d(X) V(g) f=\phi(X) V(g) f$ for all $f \in \mathcal{D}(\pi), g \in G$ and $X \in \mathscr{L}(G)$.

Since $\phi(X) V(g) f=V(g) \phi\left(\alpha_{g^{-1}}(X)\right) f \in \mathscr{D}(\pi)$ for all $g \in G$ and $a_{g^{-1}}(X)$ depends analytically on $g \in G$, it follows that one can obtain an estimate similar to (a) for the derivatives of $\phi(X) V(g) f$. It follows then that $V(g) f$ is a $C^{2}$-vector function on $G$. Continuing in this way one can show $V(g) f$ is a $C^{\infty}$-vector function on $G$ and $d(A) V(g) f=\phi(A) V(g) f$ for all $A \in \mathbb{Q}(G), g \in G$ and $f \in \mathscr{D}(\pi)$.

Hence, every vector $f \in \mathscr{D}(\pi)$ is a $C^{\infty}$-vector for the representation $g \rightarrow V(g)$. 
Since the representation $g \rightarrow V(g)$ has a dense set of $C^{\infty}$-vectors, this representation must be strongly continuous. Let $\phi^{\prime}$ be the standard representation of $\mathscr{Q}(G)$ associated with the representation $g \rightarrow V(g)$ in the manner described at the beginning of this section. We have that the domain of $\phi^{\prime}$ consists of the $C^{\infty}$-vectors for $g \rightarrow V(g)$ and

$$
\phi^{\prime}(A) f=\left.d(A) V(g) f\right|_{g=e}
$$

for all $f \in \mathscr{D}\left(\phi^{\prime}\right)$ and $A \in \mathbb{Q}(G)$. Hence, $\mathscr{D}\left(\phi^{\prime}\right) \supset \mathscr{D}(\pi)=\mathscr{D}(\phi)$ and $\phi^{\prime} \supset \phi$. Since $\phi^{\prime}$ is standard, the proof of the lemma is complete.

Remark. With a little more work one can show $\phi^{*}$ of Lemma 4.4 is standard, and with quite a bit more work one can show the closure of $\phi$ is standard. However, we will not need these results in the following.

Theorem 4.5. Suppose $\pi$ is a selfadjoint representation of $\mathscr{Q}(G)$ on a Hilbert space $\mathcal{H}$. Then $\pi$ is standard if and only if $\pi$ is completely strongly positive.

Proof. It follows immediately from the definition of $Q_{m}$ that a standard representation of $\mathscr{Q}(G)$ is completely strongly positive.

Suppose then that $\pi$ is a selfadjoint completely strongly positive representation of $\mathfrak{A}(G)$ on a Hilbert space $\mathcal{H}$. We show $\pi$ is standard. We begin by showing the kernel of $\pi$ contains the kernel of $\pi_{u}$, the universal standard representation of $\mathbb{Q}(G)$, and, therefore, using $\pi$ we can define a selfadjoint representation $\phi$ of $\mathfrak{Q}_{u}=\pi_{u}(\mathfrak{Q}(G))$.

Since $\pi$ is completely strongly positive, we have $A \in Q_{m}$ (i.e., $\left\{A \delta_{i 1} \delta_{j 1}\right\} \in Q_{m}$ ) implies $\pi(A) \geq 0$. It is a property of the universal standard representation $\pi_{u}$ that $A \in Q_{m}$ if and only if $\pi_{u}(A) \geq 0$. Hence, if $\pi_{u}(A)=0$ we have $A \in Q_{m}$ and $-A \in Q_{m}$ and, therefore, $\pi(A) \geq 0$ and $\pi(-A) \geq 0$. Hence, we have $\pi_{u}(A)=0$ implies $\pi(A)=0$. Let $\alpha$ be any mapping of $\mathfrak{Q}_{u}=\pi_{u}(\mathfrak{Q}(G))$ into $\mathfrak{Q}(G)$ such that $\pi_{u}(\alpha(A))=A$ for all $A \in \mathbb{Q}_{u}$. Let $\phi$ be mapping of $\mathbb{Q}_{u}^{u}$ into operators defined on $\mathfrak{D}(\pi)$ given by $\phi(A)=$ $\pi(\alpha(A))$ for all $A \in \mathbb{Q}_{u}$. We note $\phi$ is independent of the choice of the map $a$ since if $\pi_{u}(\alpha(A))=\pi_{u}\left(\alpha^{\prime}(A)\right)=A$ then $\pi_{u}\left(\alpha(A)-\alpha^{\prime}(A)\right)=0$ and, hence, $\pi(\alpha(A))=$ $\pi\left(\alpha^{\prime}(A)\right)$. Since $\pi$ is a selfadjoint representation of $\mathscr{Q}(G)$, it follows that $\phi$ is a selfadjoint representation of $\mathbb{Q}_{u}$ on $\mathcal{H}$ with domain $\mathscr{D}(\phi)=\mathscr{D}(\pi)$. We note that $\phi\left(\pi_{u}(A)\right)=\pi(A)$ for all $A \in \mathbb{Q}(G)$.

Since $\pi$ is completely strongly positive with respect to $Q_{m}$, it follows that $\phi$ is a completely strongly positive map with respect to $Q_{m}^{\prime}$ of $\mathfrak{C}_{u}$ on $\mathscr{D}(\pi)$. Note that since $\phi$ is a representation we can consider $\phi(A)$ as an operator on $\mathscr{D}(\pi)$ as well as a bilinear form on $\mathscr{D}(\pi) \times \mathscr{D}(\pi)$ for each $A \in \mathbb{Q}(G)$, i.e. $(f, \phi(A) g)=$ $\langle f|\phi(A)| g\rangle$ for all $f, g \in \mathscr{D}(\pi)$ and $A \in \mathbb{Q}(G)$. As we have seen, $\mathfrak{Q}_{u}$ is cofinal in $B_{u}$ with respect to $Q_{m}^{\prime}$. Therefore, by Theorem 3.7 there exists an extension $\phi^{\prime}$ of $\phi$ to $B_{u}$ as a completely strongly positive map of $B_{u}$ on $\mathcal{D}(\pi)$. Let $\phi^{\prime}$ be such an extension. It follows from the Stinespring construction (see Theorem 2.4) 
that there is a closed completely strongly positive *-representation $\pi^{\prime}$ of $\mathfrak{B}_{u}$ on a Hilbert space $\mathcal{H}^{\prime}$ and a linear map $V$ of $\mathscr{D}(\pi)$ into $\mathscr{D}\left(\pi^{\prime}\right)$ such that

$$
\left\langle f\left|\phi^{\prime}(A)\right| g\right\rangle=\left(V f, \pi^{\prime}(A) V g\right)
$$

for all $f, g \in \mathscr{D}(\pi)$ and $A \in B_{u}$ and $\left\{\pi^{\prime}\left(B_{u}\right) V \mathscr{D}(\pi)\right\}$ is strongly dense in $\mathscr{D}\left(\pi^{\prime}\right)$. Let $\pi^{\prime \prime}(A)=\pi^{\prime}\left(\pi_{u}(A)\right)$ for all $A \in \mathscr{Q}(G)$. Since $\pi^{\prime}$ is completely strongly positive and, therefore, order preserving, it follows from Lemma 4.4 that $\pi$ " has a standard extension $\pi_{1}$. Suppose $\pi_{1}$ is a standard extension of $\pi^{\prime \prime}$, i.e. $\pi_{1} \supset \pi^{\prime \prime}$.

We will show that $\pi$ is unitarily equivalent to a subrepresentation of $\pi_{1}$. Now we have $V \mathscr{D}(\pi) \subset \mathscr{D}\left(\pi^{\prime}\right)=\mathscr{D}\left(\pi^{\prime \prime}\right) \subset \mathscr{D}\left(\pi_{1}\right)$. For $f, g \in \mathscr{D}(\pi)$ and $A \in \mathscr{Q}(G)$ we have

$$
\begin{aligned}
(f, \pi(A) g) & =\left\langle f\left|\phi\left(\pi_{u}(A)\right)\right| g\right\rangle=\left\langle f\left|\phi^{\prime}\left(\pi_{u}(A)\right)\right| g\right\rangle \\
& =\left(V f, \pi^{\prime}\left(\pi_{u}(A)\right) V_{g}\right)=\left(V f, \pi^{\prime \prime}(A) V g\right)=\left(V f, \pi_{1}(A) V g\right)
\end{aligned}
$$

where the first equality follows from the definition of $\phi$, the second from the fact that $\phi^{\prime}$ extends $\phi$, the third from equation (a), the fourth from the definition of $\pi$ " and the fifth from the fact that $\pi_{1} \supset \pi^{\prime \prime}$. Hence, we have

$$
(f, \pi(A) g)=\left(V f, \pi_{1}(A) V g\right)
$$

for all $f, g \in \mathscr{D}(\pi)$ and $A \in \mathbb{Q}(G)$.

Setting $A=I$, we find $V$ is isometric. Using equation (b) one easily computes that $\left\|\pi_{1}(A) V f-V \pi(A) f\right\|^{2}=0$ for all $f \in \mathscr{D}(\pi)$ and $A \in \mathbb{Q}(G)$. Hence, it follows that $\{V \mathscr{D}(\pi)\}$ is an invariant subspace for $\pi_{1}$ and $\pi_{1} \mid\{V \mathscr{D}(\pi)\}$ is unitarily equivalent to $\pi$. Since $\pi$ is selfadjoint, $\pi_{1} \mid\{V D(\pi)\}$ is selfadjoint. Since $\pi_{1} \mid\{V \mathscr{D}(\pi)\}$ is a selfadjoint subrepresentation of a standard representation of $\mathbb{Q}(G)$, it follows from Lemma 4.2 that $\pi_{1} \mid\{V \mathscr{D}(\pi)\}$ is standard. Hence, $\pi$ is unitarily equivalent to a standard representation of $\mathscr{Q}(G)$ and, hence, $\pi$ is standard. This completes the proof of the theorem.

V. Centrally positive operators. In [4] Nelson constructed an example of two essentially selfadjoint operators $A$ and $B$ defined on a common dense invariant domain $\mathcal{D}$ such that $A$ and $B$ commute on $D$ but the spectral projections of $\bar{A}$ and $\bar{B}$ fail to commute. It follows from Nelson's example that for a selfadjoint representation $\pi$ of ${ }^{*}$-algebra $\mathfrak{Q}$ and a hermitian element $A$ in the center of $\mathcal{Q}, \overline{\pi(A)}$ need not be affiliated with $\pi(\mathscr{Q})^{\prime}$. In this section we give a necessary and sufficient condition that $\overline{\pi(A)}$ be affiliated with $\pi(\mathfrak{Q})^{\prime}$ where $A=A^{*} \epsilon \mathbb{Q}$ and $\pi$ is a selfadjoint representation of $\mathfrak{Q}$ on a Hilbert space. The condition is expressed in terms of an order structure on $M(\mathfrak{Q})$. This result is then used to characterize standard representations of commutative *-algebras in terms of an order structure.

From now until the end of the proof of Theorem 5.3 we let $\mathfrak{Q}$ denote an arbitrary *-algebra with unit. Let $Q_{0 m}$ be the minimal algebraically admissible cone in 
$M(\mathscr{Q})$, i.e. $\left\{A_{i j}\right\}=\left\{A_{i j}\right\}^{*} \in Q_{0 m}$ if and only if

$$
A_{i j}=\sum_{k=1}^{n} \sum_{l=1}^{\infty} B_{l i}^{(k)^{*}} B_{l j}^{(k)}
$$

with $\left\{B_{i j}^{(k)}\right\} \in M(\mathfrak{Q})$ for $k=1, \cdots, n$. We have $\left\{A_{i j}\right\} \in Q_{0 m}$ if and only if $\left\{A_{i j}\right\}$ is a finite sum of positive terms $\left\{B_{i j}^{(k)}\right\} *\left\{B_{i j}^{(k)}\right\}$.

Definition 5.1. Suppose $\pi$ is a ${ }^{*}$-representation of a ${ }^{*}$-algebra $\mathfrak{A}$ on a Hilbert space $\mathcal{H}$. We say a hermitian operator $\pi\left(A_{0}\right)$ with $A_{0}=A_{0}^{*} \in \mathbb{Q}$ is centrally positive if the following is true for all $m=1,2, \ldots$. If $\left\{A_{i j}^{(k)}\right\}=\left\{A_{i j}^{(k)}\right\}^{*} \in M(\mathfrak{Q})$ for $k=0,1, \ldots, m$ and

for all real $\lambda$ then

$$
\left\{\sum_{k=0}^{m} \lambda^{k} A_{i j}^{(k)}\right\}=\left\{A(\lambda)_{i j}\right\} \in Q_{0 m}
$$

$$
\sum_{k=0}^{m} \sum_{i j=1}^{\infty}\left(f_{i}, \pi\left(A_{0}^{k}\right) \pi\left(A_{i j}^{(k)}\right) f_{j}\right) \geq 0
$$

for all $f_{i} \in \mathscr{D}(\pi)$ for $i=1,2, \ldots$.

More informally, $\pi\left(A_{0}\right)$ is centrally positive if $\pi$ maps pointwise positive polynomials in $A_{0}=A_{0}^{*} \in \mathbb{Q}$ with coefficients in $M(\mathfrak{Q})$ into positive elements of $M(\pi(\mathfrak{Q}))$. We will show that if $\pi$ is a selfadjoint representation of a ${ }^{*}$-algebra $\mathfrak{Q}$ with unit and $A_{0}=A_{0} \in \mathfrak{Q}$ then $\overline{\pi\left(A_{0}\right)}$ is affiliated with $\pi(\mathfrak{Q})^{\prime}$ if and only if $\pi\left(A_{0}\right)$ is centrally positive.

Lemma 5.2. Suppose $\pi$ is a selfadjoint representation of a ${ }^{*}$-algebra $\mathfrak{Q}$ with unit on a Hilbert space $\mathcal{H}$. Suppose $A_{0}=A_{0}^{*} \in \mathbb{Q}$ and $\overline{\pi\left(A_{0}\right)}$ is affiliated with $\pi(\mathfrak{Q})^{\prime}$. Then $\overline{\pi\left(A_{0}\right)}$ is selfadjoint.

Proof. Suppose $\pi$ and $A_{0}$ satisfy the hypotheses of the lemma. Let $E_{+}$and $E_{-}$ be the projections onto the deficiency spaces of $\overline{\pi\left(A_{0}\right)}$, i.e. $E_{ \pm} \mathcal{H} \subset \mathcal{D}\left(\pi\left(A_{0}\right)^{*}\right)$ and $\pi\left(A_{0}\right) * E_{ \pm}= \pm i E_{ \pm}$.

Since $\pi$ is selfadjoint, $\pi(\mathfrak{Q})^{\prime}$ is a von Neumann algebra. Since $\overline{\pi\left(A_{0}\right)}$ is affiliated with $\pi(\mathfrak{Q})^{\prime}$, we have $E_{ \pm} \in \pi(\mathfrak{Q})^{\prime}$. Hence, we have $\left(f, E_{ \pm} \pi(A) g\right)=\left(\pi\left(A^{*}\right) f, E_{ \pm} g\right)$ for all $f, g \in \mathscr{D}(\pi)$ and $A \in \mathbb{Q}^{ \pm}$. In particular we have for all $f, g \in \mathscr{D}(\pi)$

$$
\begin{aligned}
\left(f, E_{+} g\right) & =-i\left(f, \pi\left(A_{0}\right)^{*} E_{+} g\right)=-i\left(\pi\left(A_{0}\right) f, E_{+} g\right) \\
& =-i\left(E_{+} f, \pi\left(A_{0}\right) g\right)=-i\left(\pi\left(A_{0}\right)^{*} E_{+} f, g\right)=-\left(E_{+} f, g\right)=-\left(f, E_{+} g\right) .
\end{aligned}
$$

Hence, $\left(f, E_{+} g\right)=0$ for all $f, g \in \mathscr{D}(\pi)$. Since $\mathscr{D}(\pi)$ is dense in $\mathcal{H}$, we have $E_{+}=0$. A similar computation shows $E_{-}=0$. Hence, the deficiency spaces of $\overline{\pi\left(A_{0}\right)}$ are both null spaces so $\overline{\pi\left(A_{0}\right)}$ is selfadjoint. This completes the proof of the lemma. 
Theorem 5.3. Suppose $\pi$ is a selfadjoint representation of $a^{*}$-algebra $\mathfrak{Q}$ with unit on a Hilbert space $\mathcal{H}$. Suppose $A_{0}=A_{0}^{*} \in \mathbb{Q}$. Then $\overline{\pi\left(A_{0}\right)}$ is affiliated with $\pi(\mathfrak{Q})^{\prime}$ if and only if $\pi\left(A_{0}\right)$ is centrally positive.

Proof. Suppose $\pi$ is a selfadjoint representation of $\mathbb{Q}$ on a Hilbert space $\mathcal{H}$ and $A_{0}=A_{0}^{*} \in \mathbb{Q}$. First we assume $\overline{\pi\left(A_{0}\right)}$ is affiliated with $\pi(\mathfrak{Q})^{\prime}$. Then, from Lemma 5.2, $\pi\left(A_{0}\right)$ is selfadjoint. Let $\{E(\lambda) ;-\infty<\lambda+\infty\}$ be the spectral resolution of $\overline{\pi\left(A_{0}\right)}$. Since $\overline{\pi\left(A_{0}\right)}$ is affiliated with $\pi(\mathfrak{Q})^{\prime}$, we have $E(\lambda) \in \pi(\mathfrak{Q})^{\prime}$ for all real $\lambda$. Suppose $m$ is a positive integer and $\left\{A_{i j}^{(k)}\right\}=\left\{A_{i j}^{(k)}\right\}^{*} \in M(\mathfrak{Q})$ for $k=0,1, \ldots, m$ such that

$$
\left\{\sum_{k=0}^{m} \lambda^{k} A_{i j}^{(k)}\right\}=\left\{A(\lambda)_{i j}\right\} \in Q_{0 m}
$$

for all real $\lambda$. Let $r$ be the smallest integer so that $A_{i j}^{(k)}=0$ if $i>r$ or $j>r$ for $k=0,1, \cdots, m$. Suppose $f_{i} \in \mathscr{D}(\pi)$ for $i=1, \cdots, r$. We have by the spectral theorem

$$
\begin{aligned}
\sum_{k=0}^{m} \sum_{i j=1}^{r}\left(f_{i}, \pi\left(A_{0}^{k}\right) \pi\left(A_{i j}^{(k)}\right) f_{j}\right) & \\
& =\sum_{k=0}^{m} \int_{-\infty}^{\infty} \lambda^{k} d\left\{\sum_{i j=1}^{r}\left(f_{i}, E(\lambda) \pi\left(A_{i j}^{(k)}\right) f_{j}\right)\right\} .
\end{aligned}
$$

The above integral can be approximated to arbitrary accuracy by Riemann sums of the form

$$
\begin{gathered}
\sum_{l=1}^{p} \sum_{k=0}^{m} \sum_{i j=1}^{r}\left(\hat{\lambda}_{l}\right)^{k}\left(f_{i},\left(E\left(\lambda_{l}\right)-E\left(\lambda_{l-1}\right)\right) \pi\left(A_{i j}^{(k)}\right) f_{j}\right) \\
=\sum_{l=1}^{p} \sum_{k=0}^{m} \sum_{i j=1}^{r}\left(\hat{\lambda}_{l}\right)^{k}\left(f_{i l}, \pi\left(A_{i j}^{(k)}\right) f_{j l}\right)=\sum_{l=1}^{p} a_{l}
\end{gathered}
$$

where $f_{i l}=\left(E\left(\lambda_{l}\right)-E\left(\lambda_{l-1}\right)\right) f_{i} \in \mathscr{D}(\pi)$ since $E\left(\lambda_{l}\right)-E\left(\lambda_{l-1}\right)$ is a projection in $\pi(\mathfrak{Q})^{\prime}$ and $\hat{\lambda}_{l}$ is chosen in the interval $\left[\lambda_{l-1}, \lambda_{l}\right]$. We have $\left\{\Sigma_{k=0}^{m}\left(\hat{\lambda}_{l}\right)^{k} A_{i j}^{(k)}\right\}=$ $\left\{A\left(\hat{X}_{l}\right)_{i j}\right\} \in Q_{0 m}$ for $l=1, \ldots, p$. Since $Q_{0 m}$ is the minimal algebraically. admissible cone in $M(\mathfrak{Q})$ and $\pi$ is a ${ }^{*}$-representation of $\mathfrak{Q}$ and, therefore, completely positive (completely strongly positive with respect to $Q_{0 m}$ ), we have

$$
\left.a_{l}=\sum_{k=0}^{m} \sum_{i j=1}^{r}(\hat{\lambda})_{l}\right)^{k}\left(f_{i l}, \pi\left(A_{i j}^{(k)}\right) f_{j l}\right) \geq 0
$$

for $l=1, \ldots, p$. Since all of the approximating Riemann sums are positive, we have 


$$
\sum_{k=0}^{m} \sum_{i j=1}^{r}\left(f_{i}, \pi\left(A_{0}^{k}\right) \pi\left(A_{i j}^{(k)}\right) f_{j}\right) \geq 0 .
$$

Hence, $\pi\left(A_{0}\right)$ is centrally positive.

Next, suppose $\pi\left(A_{0}\right)$ is centrally positive. We will show $\overline{\pi\left(A_{0}\right)}$ is affiliated with $\pi(\mathfrak{C})^{\prime}$. We begin by showing $\pi\left(A A_{0}-A_{0} A\right)=0$ for all $A \in \mathfrak{Q}$. To this end suppose $A=A^{*} \in \mathbb{Q}$. Clearly $\lambda^{2} A^{2}+2 \lambda A+I=(\lambda A+I) *(\lambda A+I) \in Q_{0 m}$ for all real $\lambda$. Since $\pi\left(A_{0}\right)$ is centrally positive, we have

$$
\left(f, \pi\left(A_{0}^{2} A^{2}+2 A_{0} A+I\right) f\right) \geq 0, \quad\left(f, \pi\left(A_{0}^{2} A^{2}-2 A_{0} A+I\right) f\right) \geq 0,
$$

for all $f \in \mathscr{D}(\pi)$. Subtracting these equations we obtain that $\left(f, \pi\left(A_{0} A\right) f\right)$ is real for all $f \in \mathscr{D}(\pi)$ and hence $\pi\left(A_{0} A\right)$ is hermitian. Hence, we have $\pi\left(A_{0} A\right)=$ $\pi\left(\left(A_{0} A\right)^{*}\right)=\pi\left(A^{*} A_{0}^{*}\right)=\pi\left(A A_{0}\right)$ for all $A=A^{*} \in \mathbb{Q}$. By linearity it follows that $\pi\left(A_{0} A\right)=\pi\left(A A_{0}\right)$ for all $A \in \mathbb{Q}$.

Let $B_{1}$ be the *algebra of all mappings $\lambda \rightarrow F(\lambda)$ of the real line into $\mathfrak{Q}$ of the form $F(\lambda)=\sum_{i=1}^{n} q_{i}(\lambda) A_{i}$ with $A_{i} \in \mathbb{Q}$ and $q_{i}(\lambda)$ a continuous polynomially bounded function of $\lambda$ for $i=1, \ldots, n$ and $n=1,2, \ldots$. The algebraic operations in $B_{1}$ are defined to be the pointwise operators, i.e. if $F_{1}, F_{2} \in B_{1}$ and $a$ is a complex number then

$$
\begin{aligned}
\left(\alpha F_{1}+F_{2}\right)(\lambda) & =a F_{1}(\lambda)+F_{2}(\lambda), \\
\left(F_{1} F_{2}\right)(\lambda) & =F_{1}(\lambda) F_{2}(\lambda), \\
F_{1}^{*}(\lambda) & =F_{1}(\lambda)^{*},
\end{aligned}
$$

for all real $\lambda$. We define the algebraically admissible cone $Q_{m}$ in $B_{1}$ by the statement: $\left\{F_{i j}\right\}=\left\{F_{i j}\right\}^{*} \in Q_{m}$ if and only if $\left\{F_{i j}(\lambda)\right\} \in Q_{0 m}$ for each real $\lambda$. One can easily check $Q_{m}$ is an algebraically admissible cone.

Let $\mathfrak{C}_{1}$ be the *-subalgebra of $B_{1}$ of all polynomials, i.e. $\mathfrak{Q}_{1}$ consists of all $F$ of the form $F(\lambda)=\sum_{k=0}^{m} \lambda^{k} A_{k}$ with $A_{k} \in \mathbb{Q}$ for $k=0,1, \ldots, m$ and $m=1,2, \ldots$. It is easily seen that $Q_{1}$ is cofinal in $B_{1}$ with respect to $Q_{m}$. In fact, suppose $F=F^{*} \in B_{1}$. Then $F$ can be expressed in the form $F(\lambda)=\sum_{i=1}^{n} q_{i}(\lambda) A_{i}$, with $A_{i}=A_{i}^{*} \in \mathbb{Q}$ and $q_{i}$ real polynomially bounded functions. Let $p_{i}$ be real polynomials such that $p_{i}(\lambda) \geq\left|q_{i}(\lambda)\right|$ for all real $\lambda$. Let $G(\lambda)=\sum_{i=1}^{n} 1 / 2 p_{i}(\lambda)\left(A_{i}^{2}+l\right)$. Clearly, we have $G \in \mathbb{Q}_{1}, G \in Q_{m}$ and $G-F \in Q_{m}$. Hence, $\mathbb{Q}_{1}$ is cofinal in $B_{1}$.

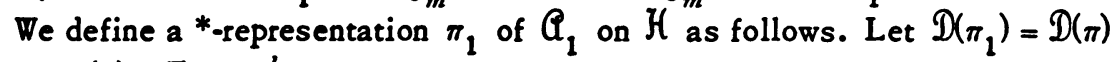
and for $F(\lambda)=\Sigma_{k=0}^{m} \lambda^{k} A_{k}$ we define

$$
\pi_{1}(F)=\pi\left(\sum_{k=0}^{m} A_{0}^{k} A_{k}\right)
$$


Since $\pi\left(A_{0} A\right)=\pi\left(A A_{0}\right)$ for all $A \in \mathbb{Q}$, one can easily check that $\pi_{1}$ is a *-representation of $\mathfrak{Q}_{1}$ on $\mathcal{H}$.

Since $\pi\left(A_{0}\right)$ is centrally positive, it follows directly from the way $Q_{m}$ was defined that $\pi_{1}$ is completely strongly positive with respect to $Q_{m}$. Since $Q_{1}$ is cofinal in $B_{1}$, it follows from Theorem 3.7 that $\pi_{1}$ has an extension $\phi$ as a completely strongly positive map of $\mathscr{B}_{1}$ on $\mathscr{D}\left(\pi_{1}\right)=\mathscr{D}(\pi)$. Let $\phi$ be such an extension.

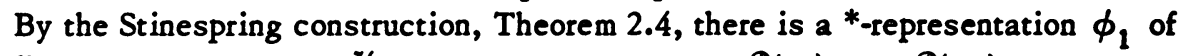
$B_{1}$ on a Hilbert space $H_{1}$ and a linear map $V$ of $D\left(\pi_{1}\right)$ into $\mathscr{D}\left(\phi_{1}\right)$ such that

$$
\langle f|\phi(A)| g\rangle=\left(V f, \phi_{1}(A) V g\right)
$$

for all $A \in B_{1}$ and $f, g \in \mathscr{D}\left(\pi_{1}\right)$ and $\left\{\phi_{1}\left(B_{1}\right) V \mathscr{D}\left(\pi_{1}\right)\right\}$ is strongly dense in $\mathscr{D}\left(\phi_{1}\right)$.

Let $\pi_{2}(A)=\phi_{1}(A)$ for all $A \in \mathbb{Q}_{1}$ and $\mathscr{D}\left(\pi_{2}\right)=\mathscr{D}\left(\phi_{1}\right)$, i.e. $\pi_{2}=\phi_{1} \mid \mathfrak{Q}_{1}$. We have $V \mathscr{D}\left(\pi_{1}\right) \subset \mathscr{D}\left(\phi_{1}\right)=\mathscr{D}\left(\pi_{2}\right)$ and

$$
\left(V f, \pi_{2}(A) V g\right)=\left(V f, \phi_{1}(A) V g\right)=\langle f|\phi(A)| g\rangle=\left\langle f\left|\pi_{1}(A)\right| g\right\rangle=\left(f, \pi_{1}(A) g\right)
$$

for all $f, g \in \mathscr{D}\left(\pi_{1}\right)$ and $A \in \mathbb{Q}_{1}$, where the first equality follows from the fact that $\pi_{2}=\phi_{1} \mid \mathfrak{Q}_{1}$, the second follows from equation (a), the third follows from the fact that $\pi_{1}=\phi \mid Q_{1}$ and the fourth follows from the fact that $\left\langle f\left|\pi_{1}(A)\right| g\right\rangle$ is the bilinear form derived from the operator $\pi_{1}(A)$. Hence, we have

$$
\left(V f, \pi_{2}(A) V g\right)=\left(f, \pi_{1}(A)_{g}\right)
$$

for all $f, g \in \mathscr{D}\left(\pi_{1}\right)$ and $A \in \mathbb{Q}_{1}$.

Setting $A=I$ in equation (b) we find $V$ is isometric. Using equation (b) one can compute that $\left\|\pi_{2}(A) V f-V \pi_{1}(A) f\right\|^{2}=0$ for all $f \in \mathscr{D}\left(\pi_{1}\right)$ and $A \in \mathcal{Q}_{1}$. Hence, it follows that $\{V \mathscr{D}(\pi)\}$ is an invariant subspace for $\pi_{2}$ and $\pi_{2} \mid\left\{V \mathscr{D}\left(\pi_{1}\right)\right\}$ is unitarily equivalent to $\pi_{1}$, i.e. $\pi_{2}(A) V f=V \pi_{1}(A) f$ for all $f \in \mathscr{D}\left(\pi_{1}\right)$.

Since $V$ is an isometric mapping of $\mathscr{D}\left(\pi_{1}\right)$ into $D\left(\pi_{2}\right), V$ has a unique extension to a linear isometry of $\mathcal{H}$ into $\mathcal{H}_{1}$. We also denote this extension by $V$. Let $G(\lambda)=(\lambda+i) I$ and $G^{-1}(\lambda)=(\lambda+i)^{-1} I$ for all real $\lambda$ where $G \in Q_{1} \subset B_{1}$ and $G^{-1} \in B_{1}$ and both $G$ and $G^{-1}$ are in the center of $B_{1}$. We have $I-\left(G^{-1}\right) * G^{-1} \in Q_{m}$ and, hence, $\phi_{1}\left(G^{-1}\right)$ is a bounded operator (in fact, we have $\left.\left\|\phi_{1}\left(G^{-1}\right)\right\| \leq 1\right)$. Let $C=V^{*} \phi_{1}\left(G^{-1}\right) V$. We show $C \in \pi_{1}\left(\mathbb{Q}_{1}\right)^{\prime}$ and $\left(f, C \pi_{1}(G) g\right)=(f, g)$ for all $f, g \in \mathscr{D}\left(\pi_{1}\right)$. For $f, g \in \mathcal{D}\left(\pi_{1}\right)$ we have

$$
\begin{aligned}
\left(f, C \pi_{1}(A)_{g}\right) & =\left(f, V^{*} \phi_{1}\left(G^{-1}\right) V \pi_{1}(A) g\right)=\left(V f, \phi_{1}\left(G^{-1}\right) \pi_{2}(A) V g\right) \\
& =\left(V f, \phi_{1}\left(G^{-1}\right) \phi_{1}(A) V g\right)=\left(V f, \phi_{1}(A) \phi_{1}\left(G^{-1}\right) V g\right) \\
& =\left(\pi_{2}\left(A^{*}\right) V f, \phi_{1}\left(G^{-1}\right) V g\right)=\left(V \pi_{1}\left(A^{*}\right) f, \phi_{1}\left(G^{-1}\right) V g\right) \\
& =\left(\pi_{1}\left(A^{*}\right) f, C_{g}\right)
\end{aligned}
$$


and

$$
\begin{aligned}
\left(f, C \pi_{1}(G)_{g}\right) & =\left(f, V^{*} \phi_{1}\left(G^{-1}\right) V \pi_{1}(G) g\right)=\left(V f, \phi_{1}\left(G^{-1}\right) \pi_{2}(G) V g\right) \\
& =\left(V f, \phi_{1}\left(G^{-1}\right) \phi_{1}(G) V g\right)=(V f, V g)=(f, g) .
\end{aligned}
$$

If $F(\lambda)=A$ for all real $\lambda$ then $\pi_{1}(F)=\pi(A)$. Since $C \in \pi_{1}\left(Q_{1}\right)^{\prime}$, it follows that $C \in \pi(\mathfrak{Q})^{\prime}$. Since $\pi_{1}(G)=\pi\left(A_{0}+i l\right)$, it follows that

$$
\left(f, C \pi\left(A_{0}+i I\right)_{g}\right)=(f, g)
$$

for all $f, g \in \mathscr{D}(\pi)$. Since $C \in \pi(\mathfrak{Q})^{\prime}$ and $\pi$ is selfadjoint, we have $C \mathscr{D}(\pi) \subset \mathscr{D}(\pi)$ and

$$
C \pi\left(A_{0}+i l\right) g=\pi\left(A_{0}+i l\right) C g=g
$$

for all $g \in \mathscr{D}(\pi)$. It follows from this equation that $\pi\left(A_{0}+i I\right)$ has dense range in $\mathcal{H}$ and, hence, $\overline{\pi\left(A_{0}+i l\right)}$ has range all of $\mathcal{H}$. Hence, $\overline{\pi\left(A_{0}+i l\right)^{-1}}$ exists as a bounded operator.

From the above equation we have

$$
C_{g}=\overline{\pi\left(A_{0}+i I\right)^{-1}} \pi\left(A_{0}+i I\right) C_{g}=\overline{\pi\left(A_{0}+i I\right)^{-1}} g
$$

for all $g \in \mathscr{D}(\pi)$. Since $C$ and $\overline{\pi\left(A_{0}+i I\right)^{-1}}$ are bounded and $\mathcal{D}(\pi)$ is dense in $\mathcal{H}$, we have $C=\overline{\pi\left(A_{0}+i I\right)^{-1}}$. Since $C \in \pi(\mathfrak{Q})^{\prime}$, we have $\overline{\pi\left(A_{0}+i l\right)}$ is affiliated with $\pi(\mathfrak{Q})^{\prime}$. Hence, $\frac{0}{\pi\left(A_{0}\right)}$ is affiliated with $\pi(\mathfrak{Q})^{\prime}$. This completes the proof of the theorem.

Theorem 5.3 can be used to characterize standard representations of commutative *-algebras in terms of an order structure. The details are as follows.

In [ 5 ] it was shown that for a selfadjoint representation $\pi$ of a commutative *-algebra $\mathfrak{Q}$ with unit on a Hilbert space $\mathcal{H}$ the following statements are equivalent:

(i) $\pi(A)^{*}=\overline{\pi(A)}$ for all hermitian $A \in \mathbb{Q}$.

(ii) $\pi(A)^{*}=\overline{\pi\left(A^{*}\right)}$ for all $A \in \mathfrak{Q}$.

(iii) $\pi(\mathfrak{Q}) "$ is commutative.

We say a *-representation $\pi$ of a commutative *-algebra $\mathfrak{C}$ with unit is standard if $\pi$ is selfadjoint and satisfies any one and, therefore, all of the above conditions (i), (ii) and (iii).

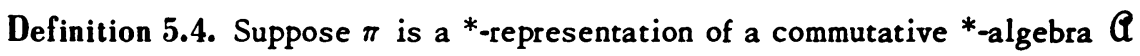
with unit on a Hilbert space $\mathcal{H}$. We say $\pi$ is completely strongly positive if the following statement is true for all $n, m=1,2, \ldots$.

Suppose $P_{i j}\left(x_{1}, \ldots, x_{n}\right)$ are for each $i, j=1, \ldots, n$ polynomials in the $m$ real variables $x_{1}, \cdots, x_{m}$ with complex coefficients such that for each point $\left(x_{1}, \ldots, x_{n}\right) \in R^{m}$ the $(n \times n)$-matrix $a_{i j}=P_{i j}\left(x_{1}, \ldots, x_{n}\right)$ is positive (i.e. $\sum_{i j=1}^{n} a_{i j} \bar{c}_{i} c_{j} \geq 0$ for all complex numbers $c_{i}$ ). Suppose $A_{i}=A_{i}^{*} \in \mathbb{Q}$ for $i=1, \ldots, n$ and $P_{i j}=P_{i j}\left(A_{1}, \ldots, A_{n}\right)$ are the polynomials $P_{i j}\left(x_{1}, \ldots, x_{n}\right)$ composed with the $A_{i}$ replacing the $x_{i}$ and suppose $f_{i} \in \mathscr{D}(\pi), i=1, \ldots, n$. Then it is true that $\sum_{i j=1}^{n}\left(f_{i}, \pi\left(P_{i j}\right) f_{j}\right) \geq 0$. 
Although the above definition is somewhat lengthy the basic idea is simple. A completely strongly positive *-representation of a commutative *-algebra is one which maps positive matrices of elements of $\mathbb{Q}$ into positive matrices of operators on $\mathfrak{D}(\pi)$.

Corollary 5.5. Suppose $B$ is the free commutative *-algebra on sermitian generators, $C_{1}, C_{2}, \cdots, C_{s}$, i.e. $B$ consists of all polynomials $P\left(C_{1}, \ldots, C_{s}\right)$ in $C_{1}, \ldots, C_{s}$. Suppose $\pi$ is a selfadjoint representation of $B$ on a Hilbert space $\mathcal{H}$. Then $\pi$ is standard if and only if $\pi$ is completely strongly positive.

Proof. First suppose $\pi$ is a standard representation of $B$ on a Hilbert space H. Suppose $n, m \geq 1$ are integers and $P_{i j}\left(x_{1}, \ldots, x_{m}\right)$ are, for each $i, j=1, \ldots, n$, polynomials satisfying the statement in Definition 5.4. Suppose $A_{i}=A_{i}^{*} \in \mathbb{Q}$ for $i=1, \ldots, m$. Since $\pi$ is standard, we have $\overline{\pi\left(A_{i}\right)}$ is selfadjoint for $i=1, \ldots, m$. Let $\left\{E_{i}(\lambda),-\infty<\lambda<+\infty\right\}$ be the spectral resolution of $\overline{\pi\left(A_{i}\right)}$. Since $\pi(\mathscr{Q})$ " is commutative and $E_{i}(\lambda) \in \pi(\mathfrak{Q})^{\prime \prime}$, we have that $\left\{E_{i}(\lambda) ;-\infty<\lambda<\infty, i=1, \ldots, m\right\}$ forms a commuting set of operators. Let $E\left(\lambda_{1}, \cdots, \lambda_{m}\right)=E_{1}\left(\lambda_{1}\right) E_{2}\left(\lambda_{2}\right) \cdots E_{m}\left(\lambda_{m}\right)$ be the joint spectral resolution for the $\overline{\pi\left(A_{i}\right)}, i=1, \ldots, m$. Then, if $P_{i j}=$ $P_{i j}\left(A_{1}, \cdots, A_{m}\right)$ and $f_{i} \in \mathscr{D}(\pi)$ for $i, j=1, \cdots, n$ then it follows from the spectral calculus that

$$
\sum_{i j=1}^{n}\left(f_{i}, \pi\left(P_{i j}\right) f_{j}\right)=\int \sum_{i j=1}^{n} P_{i j}\left(\lambda_{1} \cdots \lambda_{m}\right) d\left(f_{i}, E\left(\lambda_{1} \cdots \lambda_{m}\right) f_{j}\right) .
$$

Since $d\left(f_{i}, E\left(\lambda_{1}, \ldots, \lambda_{m}\right) f_{j}\right)$ is a positive matrix valued measure on $R^{m}$ and $P_{i j}\left(\lambda_{1} \cdots \lambda_{m}\right)$ is a positive matrix valued function on $R^{m}$, it follows that the above quantity is nonnegative. Hence, $\pi$ is completely strongly positive.

Next, suppose $\pi$ is a selfadjoint completely strongly positive representation of $\mathfrak{B}$ on $\mathcal{H}$. It follows directly from the definitions of centrally positive operators and completely strongly positive representations that $\pi\left(C_{i}\right)$ is centrally positive for each generator $C_{i}=C_{i}^{*} \in B$ for $i=1, \ldots, s$. Then it follows from Theorem 5.3 that $\overline{\pi\left(C_{i}\right)}$ is affiliated with $\pi(\mathfrak{Q})^{\prime}$ and $\overline{\pi\left(C_{i}\right)}$ is selfadjoint for each $i=1, \ldots, s$. Let $\left\{E_{i}(\lambda),-\infty<\lambda<+\infty\right\}$ be the spectral resolution of $\overline{\pi\left(C_{i}\right)}$ for $i=1, \ldots, s$. Since $\frac{i}{\pi\left(C_{i}\right)}$ is affiliated both with $\pi(\mathfrak{Q})^{\prime}$ and $\pi(\mathfrak{Q})^{\prime \prime}$, it follows that $E_{i}(\lambda) \in \pi(\mathfrak{Q})^{\prime \prime}$ $\cap \pi(\mathscr{Q})^{\prime}$ for all $i=1, \ldots, s$ and all real $\lambda$. Since the $C_{i}$ generate $B$, the $E_{i}(\lambda)$ generate $\pi(\mathfrak{Q})^{\prime \prime}$ as a von Neumann algebra. Hence, $\pi(\mathfrak{Q})^{\prime \prime}$ is generated by a commuting set of operators. Hence, $\pi(\mathfrak{Q})^{\prime \prime}$ is commutative and $\pi$ is standard. This completes the proof of the corollary.

We now extend this corollary to all commutative *algebras with unit.

Theorem 5.6. Suppose $\mathfrak{A}$ is an arbitrary commutative *-algebra with unit and $\pi$ is a selfadjoint representation of $\mathfrak{A}$ on a Hilbert space $\mathcal{H}$. Then $\pi$ is standard if and only if $\pi$ is completely strongly positive. 
Proof. First suppose $\pi$ is a standard representation of $\mathfrak{A}$ on $\mathcal{H}$. Repeating the first part of the proof of Corollary 5.5 which never made use of the fact that $B$ was finitely generated we find $\pi$ is completely strongly positive.

Next, suppose $\pi$ is a selfadjoint completely strongly positive representation of $\mathfrak{Q}$ on $\mathcal{H}$. We show $\pi$ is standard.

Suppose $A_{0}=A_{0}^{*} \in \mathbb{Q}$. We show $\overline{\pi\left(A_{0}\right)}$ is affiliated with $\pi(\mathfrak{Q})^{\prime}$. Let $\mathfrak{B}_{n}$ be the free commutative ${ }^{*}$-algebra on $n+1$ hermitian generators, $C_{0}, C_{1}, \ldots, C_{n}$, i.e. $B_{n}$ consists of all polynomials in the $C_{0}, C_{1}, \cdots, C_{n}$.

Suppose $S=\left\{A_{1}, \cdots, A_{n(s)}\right\}$ is a finite set of hermitian elements $A_{i}=A_{i}^{*} \in \mathbb{Q}$ for $i=1, \cdots, n(s)$. Let $\pi_{s}$ be the ${ }^{*}$-representation of $B_{n(s)}$ on $\mathcal{H}$ such that $\mathscr{D}\left(\pi_{s}\right)=\mathscr{D}(\pi)$ and

$$
\pi_{s}\left(P\left(C_{0}, C_{1}, \ldots, C_{n(s)}\right)\right)=P\left(\pi\left(A_{0}\right), \pi\left(A_{1}\right), \ldots, \pi\left(A_{n(s)}\right)\right)
$$

for all $P \in B_{n(s)}$, i.e. $\pi_{s}$ maps each polynomial in the $C_{i}$ into the corresponding polynomial in the $\pi\left(A_{i}\right)$. Since $\pi$ is completely strongly positive, $\pi_{s}$ is completely strongly positive. As remarked in the proof of Corollary 5.5, since $\pi_{s}$ is completely strongly positive $\pi_{s}\left(A_{0}\right)$ is centrally positive. Since $\pi_{s}\left(A_{0}\right)$ is centrally positive, it follows from the proof of Theorem 5.3 that there is a bounded operator $C_{s}$ on $\mathcal{H}$ such that $C_{s} \in \pi_{s}\left(\mathbb{B}_{n(s)}\right)^{\prime}$ and

$$
\left(f, C_{s} \pi_{s}\left(C_{0}+i I\right) g\right)=(f, g)
$$

for all $f, g \in \mathscr{D}\left(\pi_{s}\right)=\mathscr{D}(\pi)$. (Recall that in the proof of Theorem 5.3 the selfadjointness of $\pi$ was not used until after $C$ was constructed.)

From the definition of $\pi_{s}$ we have

$$
\left(f, C_{s} \pi\left(A_{i}\right) g\right)=\left(\pi\left(A_{i}\right) f, C_{s} g\right) \text { and }\left(f, C_{s} \pi\left(A_{0}+i l\right) g\right)=(f, g)
$$

for all $f, g \in \mathscr{D}(\pi)$ and $A_{i}=A_{i}^{*} \in S$ for $i=0,1, \ldots, n(s)$. Let $\Re_{s}$ be the complex linear span of $\left\{A_{0}, A_{1}, \cdots, A_{n(s)}\right\}$.

Then, we have

$$
\left(f, C_{s} \pi(A) g\right)=\left(\pi\left(A^{*}\right) f, C_{s} g\right)
$$

and

$$
\left(f, C_{s} \pi\left(A_{0}+i I\right)_{g}\right)=(f, g)
$$

for all $f, g \in \mathscr{D}(\pi)$ and $A \in \mathfrak{M}$. Hence, for each finite set $S$ of hermitian elements of $\mathbb{A}$ there exists an operator $C_{s}$ on $\mathcal{H}$ such that $\left\|C_{s}\right\| \leq 1$ and $C_{s}$ satisfies (a) and (b) above. By the axiom of choice there exists a net $S \rightarrow C_{s}$ of such operators where the sets $S$ are ordered by inclusion. Since the unit ball of $\mathbb{B}(\mathcal{H})$ is compact in the weak operator topology [2, Chapter IV, $\$ 2$, Theorem 1, Corollary 3], the net $\left\{C_{s}\right\}$ has at least one cluster point in the weak topology. Let $C$ be a 
cluster point of the net $S \rightarrow C_{s}$. We claim $C \in \pi(\mathfrak{Q})^{\prime}$ and $C \pi\left(A_{0}+i I\right) f=f$ for all $f \in \mathfrak{D}(\pi)$.

Since $\left(f, C_{s} \pi\left(A_{0}+i I\right)_{g}\right)=(f, g)$ for all $f, g \in \mathscr{D}(\pi)$ and all $S$, we have $C_{s} \pi\left(A_{0}+i l\right) g=g$ for all $g \in \mathscr{D}(\pi)$ and all finite sets $S$. Since $C$ is a cluster point of the net $S \rightarrow C_{s}$, it follows that $C \pi\left(A_{0}+i I\right) g=g$ for all $g \in \mathscr{D}(\pi)$. Next suppose $A \in \mathbb{Q}, f, g \in \mathscr{D}(\pi)$ and $\epsilon>0$. Since $C$ is a weak cluster point of the net $S \rightarrow C_{s}$, there is a finite set $S$ such that $A, A^{*} \in \mathbb{M}_{s}$ and

$$
\left|\left(\pi\left(A^{*}\right) f_{0}\left(C-C_{s}\right) g_{g}\right)\right|<\epsilon / 2, \quad\left|\left(f,\left(C-C_{s}\right) \pi(A) g\right)\right|<\epsilon / 2 .
$$

Combining these inequalities with equation (a) we find

$$
\left|\left(\pi\left(A^{*}\right) f, C g\right)-(f, C \pi(A) g)\right|<\epsilon .
$$

Since $\epsilon$ is arbitrary, we have $\left(\pi\left(A^{*}\right) f, C g\right)=(f, C \pi(A) g)$ for all $f, g \in \mathscr{D}(\pi)$ and $A \in \mathbb{Q}$. Hence, $C \in \pi(\mathbb{Q})^{\prime}$ and $C \pi\left(A_{0}+i I\right) f=f$ for all $f \in \mathscr{D}(\pi)$. Since $\pi$ is selfadjoint, we have $C \mathscr{D}(\pi) \subset \mathscr{D}(\pi)$ and hence

$$
C \pi\left(A_{0}+i I\right) f=\pi\left(A_{0}+i l\right) C f
$$

for all $f \in \mathscr{D}(\pi)$. As shown in the last part of the proof of Theorem 5.3 the above equation implies $C={\overline{\pi\left(A_{0}+i I\right)^{-1}}}^{-1}$ and hence $\overline{\pi\left(A_{0}\right)}$ is affiliated with $\pi(\mathfrak{Q})^{\prime}$. By Lemma 5.2 it follows that $\overline{\pi\left(A_{0}\right)}$ is selfadjoint. Hence, we have for each hermitian $A_{0}=A_{0}^{*} \in \mathbb{Q}, \overline{\pi\left(A_{0}\right)}$ is selfadjoint. Hence, $\pi$ is standard, and this completes the proof of the theorem.

\section{REFERENCES}

1. William B. Arveson, Subalgebras of $C^{*}$-algebras, Acta. Math. 123 (1969), 141-224, MR $40 \# 6274$.

2. N. Bourbaki, Eléments de mathématiques. Fasc. XV. Livre V: Espaces vectoriels topologiques, Actualités Sci. Indust., no. 1189, Hermann, Paris, 1953. MR 14, 880.

3. N. Dunford and J. T. Schwartz, Linear operators. I: General theory, Pure and Appl. Math., vol. 7, Interscience, New York, 1958. MR 22 \#8302.

4. E. Nelson, Analytic vectors, Ann. of Math. (2) 70 (1959), 572-615. MR 21 \#5901.

5. Robert T. Powers, Selfadjoint algebras of unbounded operators, Comm. Math. Phys. 21 (1971), 85-124. MR 44 \#811.

6. W. F. Stinespring, Positive functions on $C^{*}$-algebras, Proc. Amer. Math. Soc. 6 (1955), 211-216. MR 16, 1033.

7. B. Sz.-Nagy, Extension of linear transformations in Hilbert space which extend beyond this space, Appendix to F. Riesz and B. Sz.-Nagy, Functional analysis, Ungar, New York, 1955. MR 17, 175.

DEPARTMENT OF MATHEMATICS, UNIVERSITY OF PENNSYLVANIA, PHILADELPHIA, PENNSYLVANIA 19104 